\title{
Quantum oscillations of photocurrents in HgTe quantum wells with Dirac and parabolic dispersions
}

\author{
C. Zoth, ${ }^{1}$ P. Olbrich, ${ }^{1}$ P. Vierling, ${ }^{1}$ K.-M. Dantscher, ${ }^{1}$ V. V. Bel'kov, ${ }^{2}$ M. A. Semina, ${ }^{2}$ M. M. Glazov, ${ }^{2}$ L. E. Golub, ${ }^{2}$ \\ D. A. Kozlov, ${ }^{3,4}$ Z. D. Kvon, ${ }^{3,4}$ N. N. Mikhailov, ${ }^{3}$ S. A. Dvoretsky, ${ }^{3}$ and S. D. Ganichev ${ }^{1}$ \\ ${ }^{1}$ Terahertz Center, University of Regensburg, 93040 Regensburg, Germany \\ ${ }^{2}$ Ioffe Physical-Technical Institute, Russian Academy of Sciences, 194021 St. Petersburg, Russia \\ ${ }^{3}$ A.V. Rzhanov Institute of Semiconductor Physics, Novosibirsk 630090, Russia \\ ${ }^{4}$ Novosibirsk State University, Novosibirsk 630090, Russia \\ (Received 4 July 2014; revised manuscript received 29 August 2014; published 14 November 2014)
}

\begin{abstract}
We report on the observation of magneto-oscillations of terahertz radiation induced photocurrent in $\mathrm{HgTe} / \mathrm{HgCdTe}$ quantum wells of different widths, which are characterized by a Dirac-like, inverted, and normal parabolic band structure. The photocurrent data are accompanied by measurements of photoresistance (photoconductivity), radiation transmission, as well as magnetotransport. We develop a microscopic model of a cyclotron-resonance assisted photogalvanic effect, which describes main experimental findings. We demonstrate that the quantum oscillations of the photocurrent are caused by the crossing of Fermi level by Landau levels resulting in the oscillations of spin polarization and electron mobilities in spin subbands. Theory explains a photocurrent direction reversal with the variation of magnetic field observed in experiment. We describe the photoconductivity oscillations related with the thermal suppression of the Shubnikov-de Haas effect.
\end{abstract}

DOI: 10.1103/PhysRevB.90.205415

PACS number(s): 73.21.Fg, 72.25.Fe, 78.67.De, 73.63.Hs

\section{INTRODUCTION}

The physics of relativistic Dirac fermions in semiconductors has became a topical field of condensed matter due to their unique electronic, optic, and optoelectronic properties. One can distinguish two groups of such materials, characterized by either weak spin-orbit coupling like graphene, for recent reviews see Refs. [1-3], or by rather strong spin-orbit interaction, typical for the most of topological insulators, for reviews see Refs. [4-6]. Among the representatives of the latter group, the HgTe-based crystalline structures have attracted particular attention, because they allow one to fabricate two- and three-dimensional topological insulators [7-16]. In this very system, one can obtain Dirac-like, inverted, and normal energy dispersions without changing the material [7,8,17-32]. Thus, investigating various electronic properties in HgTe-based quantum wells with different thicknesses one can address similarities and differences in phenomena excited for different types of electron energy spectra.

Here we report on the complex study of photocurrent, photoresistance, optical transmission, and electron transport in HgTe quantum wells with the thicknesses ranging 5-21 nm where possible variants of energy spectrum are realized. While the terahertz $(\mathrm{THz})$ radiation induced photogalvanic currents [33-36] in HgTe quantum wells (QWs) subjected to a classical magnetic field $\boldsymbol{B}$ are studied in detail in Refs. [19,29], our paper focuses on the observation and analysis of quantum oscillations in the cyclotron-resonance-assisted photocurrent excited by $\mathrm{THz}$ laser radiation. We show that the photocurrent quantum oscillations, similar to the de Haas-van Alphen and Shubnikov-de Haas effects, stem from the consecutive crossings of Fermi level by Landau levels, but are drastically enhanced due to the cyclotron resonance (CR). We discuss the microscopic origin of the photocurrent in all three cases of electron energy dispersion and demonstrate that it is caused by the magnetogyrotropic photogalvanic effect [37]. While the main features of the phenomena, such as $1 / B$-periodic oscillations superimposed by the $\mathrm{CR}$ resonance, are very general and the effect is of the same order of magnitude for all studied samples, strong peculiarities for Dirac fermions have been observed. Particularly, as a distinguishing feature of the linear spectrum, cyclotron resonance and quantum oscillations in the photocurrent are obtained simply by the variation of the carrier density in a QW.

\section{SAMPLES, MAGNETOTRANSPORT, DATA AND METHODS}

\section{A. Samples}

The experiments are carried out on doped (013)-oriented MBE grown $\mathrm{Hg}_{0.3} \mathrm{Cd}_{0.7} \mathrm{Te} / \mathrm{HgTe} / \mathrm{Hg}_{0.3} \mathrm{Cd}_{0.7} \mathrm{Te}$ single $\mathrm{QW}$ structures [38] with different widths, $L_{w}$, of 5, 6.6, 8, and $21 \mathrm{~nm}$, mobilities of about $10^{5} \mathrm{~cm}^{2} /(\mathrm{V} \mathrm{s})$ at $T=4.2 \mathrm{~K}$ and carrier densities $n$ in the range $5 \times 10^{10}-7.5 \times 10^{11} \mathrm{~cm}^{-2}$. In $\mathrm{HgTe}$, an increase of the QW thickness results in the qualitative change of the band structure [7,8], starting with a normal parabolic dispersion (5 nm QW), switching to Dirac cones $(6.6 \mathrm{~nm})$ and then to an inverted, close to parabolic, band structure (8 and $21 \mathrm{~nm})$. Besides the structures with pure HgTe QWs, we also studied $\mathrm{Hg}_{0.86} \mathrm{Cd}_{0.14}$ Te QWs with the same barriers but containing $14 \% \mathrm{Cd}$ in the QW layer. The most important difference of such QWs, compared to that made of pure $\mathrm{HgTe}$, is that the transition from a normal to an inverted energy spectrum is observed for wider QWs [21,27]. This fact allows us to study the same phenomena in material with inverted or noninverted band structures in QWs of the same thickness, here $L_{w}=8 \mathrm{~nm}$.

The samples are prepared in different geometries including Hall bar structures, without a gate and with a semitransparent gate, as well as square-shaped samples of $5 \times 5 \mathrm{~mm}^{2}$ size. While the square-shaped large-size structures require van der Pauw geometry for transport measurements, they are prepared in order to enable simultaneous measurements of the photoresponse and radiation transmission. The typical structure designs and the ohmic contacts positions for Hall-bar 

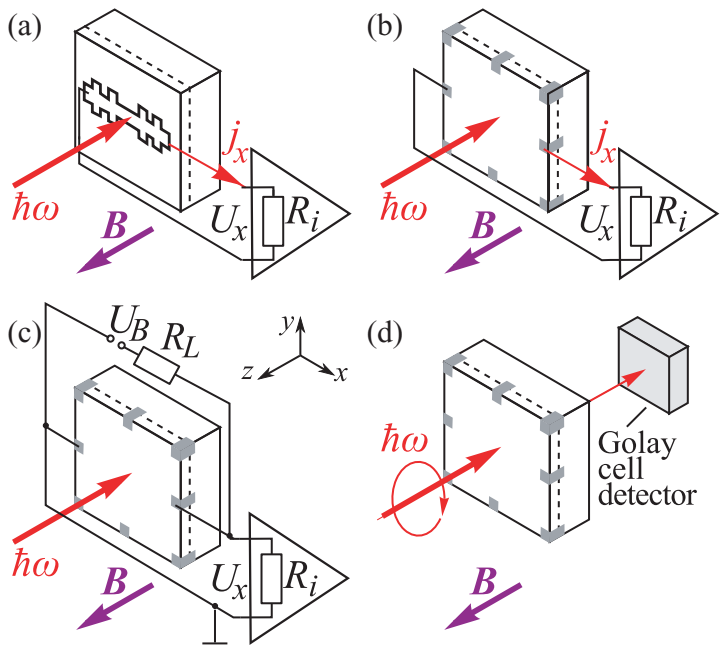

FIG. 1. (Color online) Experimental geometries of photocurrent measurements for Hall bar- and square-shaped samples in (a) and (b), respectively, for (c) photoresistance, as well as (d) transmission.

and square-shaped samples are shown in Fig. 1. Note that for the square-shaped samples eight ohmic contacts have been prepared in the middle of the edges and on the corners of the structure. For magneto-optic and magnetotransport experiments a magnetic field $\boldsymbol{B}$ up to $7 \mathrm{~T}$ is applied normal to the QW plane.

\section{B. Magnetotransport data}

In all samples well-pronounced Shubnikov-de Haas ( $\mathrm{SdH})$ oscillations of static conductivity, see dashed lines in Figs. 2-6, and quantum Hall plateaus (not shown) have been detected. Magnetotransport has been measured in a four-terminal configuration applying slowly modulated bias $(f=12 \mathrm{~Hz}, 1 \mathrm{~V})$ to the sample. The carrier densities $n$ at $4.2 \mathrm{~K}$ are given in Table I.

At high magnetic field, SdH oscillations corresponding to both even and odd filling factors and having similar amplitude are observed in all samples characterized by almost parabolic dispersion, see Figs. 2-5. This indicates that the absolute value of the Zeeman splitting $\left|\Delta_{Z}\right|$ is comparable to the energy difference between neighboring levels $\Delta E=$

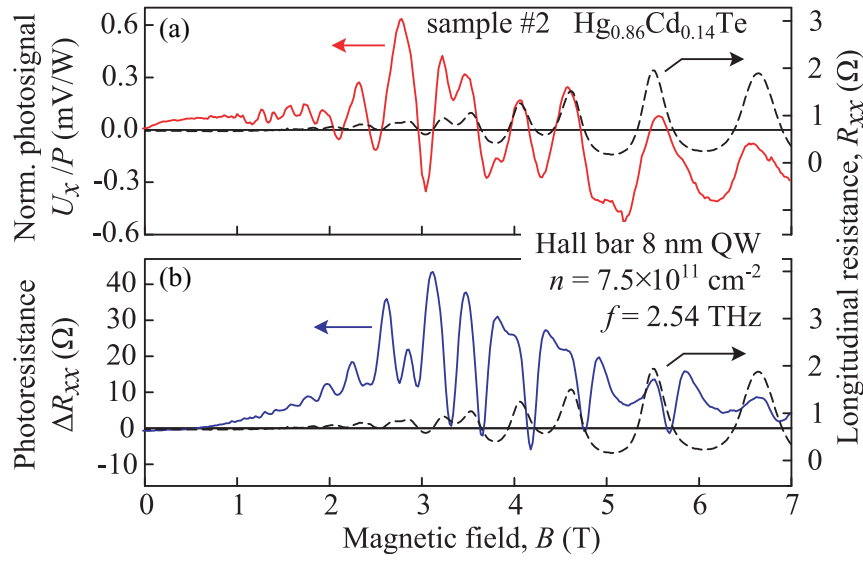

FIG. 2. (Color online) Photosignals obtained for an ungated 8-nm $\mathrm{HgCdTe}$ Hall bar sample 2 excited by linearly polarized radiation as a function of magnetic field $B$. Panel (a) shows the normalized by the radiation power photosignal $U_{x} / P$ induced in the unbiased sample, and panel (b) presents the photoresistance response $\Delta R_{x x}$ measured in the biased sample. Dashed curves show $\mathrm{SdH}$ oscillations of resistivity $R_{x x}$.

$\hbar \omega_{c}-\left|\Delta_{Z}\right|$. Here $\Delta_{Z}=g \mu_{B} B$ with $g$ being the effective electron $g$ factor, $\mu_{B}$ being the Bohr magnetron, $B=|\boldsymbol{B}|$ being the magnetic field directed along the sample normal, the cyclotron frequency $\omega_{c}=|e B| / m_{c} c$, with carrier charge $e$, speed of light $c$, and the cyclotron mass $m_{c}$. The latter is given by $m_{c}=\hbar^{2} k d k / d E$, where $k$ and $E=E(k)$ are wave vector and energy, respectively. In the particular case of linear energy dispersion characterized by a constant velocity $v_{\mathrm{DF}}$ the cyclotron mass $m_{c}$ depends on the Fermi energy $E_{\mathrm{F}}=\sqrt{2 \pi n}\left(\hbar v_{\mathrm{DF}}\right)$ as $m_{c}=E_{\mathrm{F}} / v_{\mathrm{DF}}^{2}[40]$.

Measuring the $\mathrm{SdH}$ oscillations in samples with parabolic dispersion subjected to low magnetic fields we detect either even or odd numbers of minima, depending on the electron density of the sample. This is caused by the fact that at low magnetic fields the distance between neighboring levels $\Delta E$ is smaller than the level broadening caused by the electronscattering processes. Hence, even minima are observed if the absolute value of the Zeeman splitting $\left|\Delta_{Z}\right|$ is smaller than $\Delta E$. Such a behavior is detected, e.g., for magnetic fields $B \lesssim 2.5 \mathrm{~T}$ in the Hall bar 8-nm $\mathrm{Hg}_{0.86} \mathrm{Cd}_{0.14}$ Te sample 2 having

TABLE I. Parameters of the investigated samples at $T=4.2 \mathrm{~K}$. Second and third columns show the Hg contents in quantum well and QW width. The transport scattering times $\tau_{\text {tr }}$ have been evaluated from the electron mobility and $\tau_{\mathrm{CR}}$ have been estimated from full width at half maximum of radiation transmittance measurements under the cyclotron resonance conditions. Carrier densities given for samples 3 and 6 are obtained by optical doping. For that the structures were illuminated for time $t_{\text {ill }}$ with red LED. Magnetotransport measurements carried out on gated Hall bar sample 4 show that the transport relaxation time in this sample increases with the rising carrier density as $\tau_{\text {tr }} \propto \sqrt{n}$. This result is in full agreement with the theory for systems characterized by the linear dispersion and short-range scattering; for details see Refs. [28,39].

\begin{tabular}{|c|c|c|c|c|c|c|c|}
\hline Sample & $\mathrm{Hg}(\%)$ & $L_{w}(\mathrm{~nm})$ & Design & $t_{\text {ill }}(\mathrm{s})$ & $n\left(10^{11} \mathrm{~cm}^{-2}\right)$ & $\tau_{\mathrm{CR}}(\mathrm{ps})$ & $\tau_{\text {tr }}(\mathrm{ps})$ \\
\hline 1 & 100 & 5 & square & & 2.4 & 0.29 & 0.24 \\
\hline 2 & 86 & 8 & Hall bar & & 7.5 & & 0.74 \\
\hline 3 & 100 & 6.6 & square & 80 & 1.1 & 0.44 & 0.59 \\
\hline 4 & 100 & 6.6 & gated Hall bar & & $0.5-4.5$ & & 0.82 at $n=10^{11} \mathrm{~cm}^{-2}$ \\
\hline 5 & 100 & 8 & square & & 2.4 & 0.68 & 0.65 \\
\hline 6 & 100 & 8 & square & 80 & 3.2 & 0.76 & 0.68 \\
\hline 7 & 100 & 21 & square & & 1.7 & 1.4 & 1.58 \\
\hline
\end{tabular}



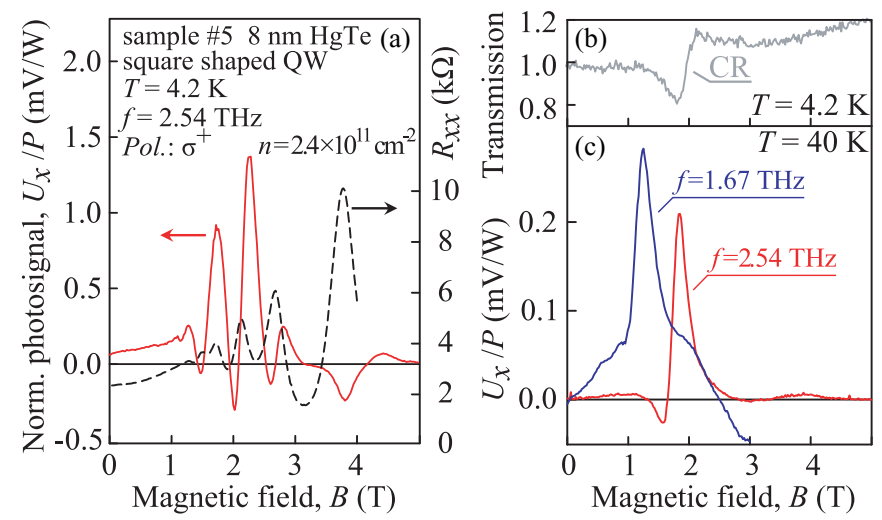

FIG. 3. (Color online) Photosignal normalized by the radiation power, $U_{x} / P$, for the 8-nm pure HgTe QW square sample 5, excited by $\sigma^{+}$radiation vs magnetic field $B$ at (a) $T=4.2 \mathrm{~K}$ and (c) $T=40 \mathrm{~K}$. Latter data are shown for two different frequencies, $2.54 \mathrm{THz}$ (red) and $1.64 \mathrm{THz}$ (blue). Panel (b) shows the change of the radiation transmission upon sweeping magnetic field. The data are obtained for $f=2.54 \mathrm{THz}$ and are given in arbitrary units. Black dashed curve in panel (a) shows $\mathrm{SdH}$ oscillations of resistivity $R_{x x}$.

high electron density $n=7.5 \times 10^{11} \mathrm{~cm}^{-2}$; see Fig. 2 . The decrease of electron density results in the lowering of the Fermi energy and, correspondingly, increase of the electron
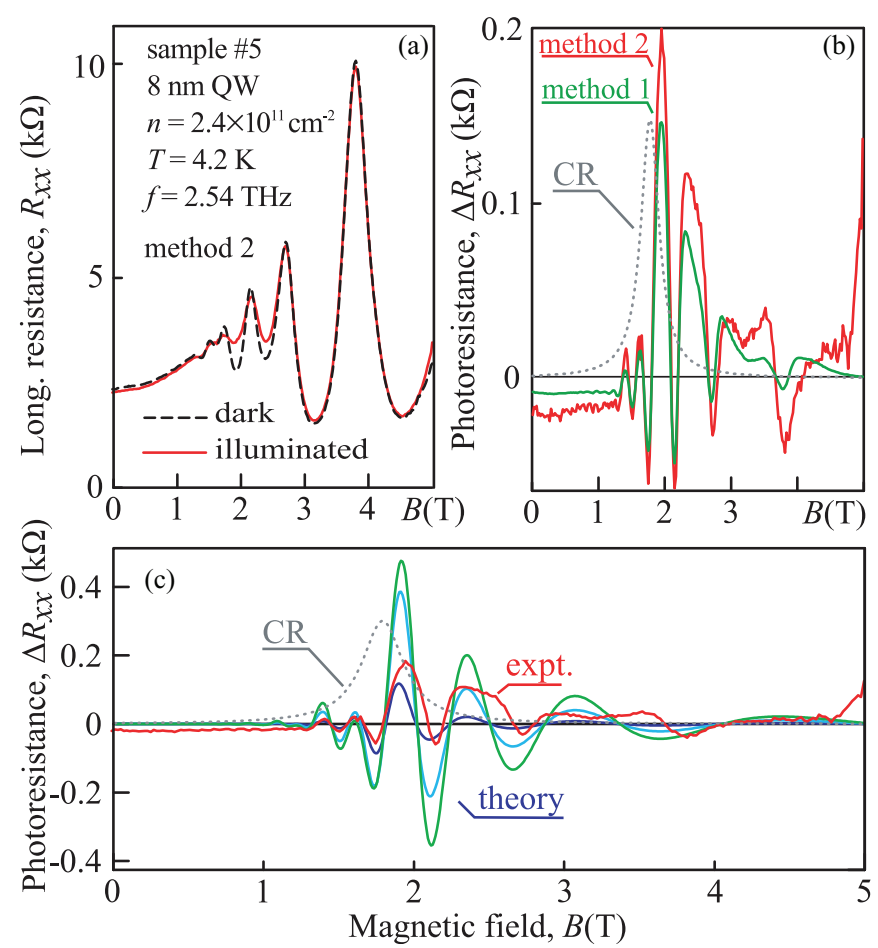

FIG. 4. (Color) Photoresistance and $\mathrm{SdH}$ data for square sample 5 with $L_{w}=8 \mathrm{~nm}$. (a) Longitudinal resistance measured with (red/solid) and without (black/dotted) cw THz radiation. (b) Green curve shows photoresistance, $\Delta R_{x x}$, for dc bias and modulated radiation (method 1), red curve shows $\Delta R_{x x}$ for ac bias and nonmodulated radiation (method 2). (c) Measured $\Delta R_{x x}$ (second method, left scale) and theoretical fits (three curves corresponding to different heating rates; arb. units). Dashed lines marked as "CR" in panels (b) and (c) show calculated cyclotron resonance absorption (arb. units).
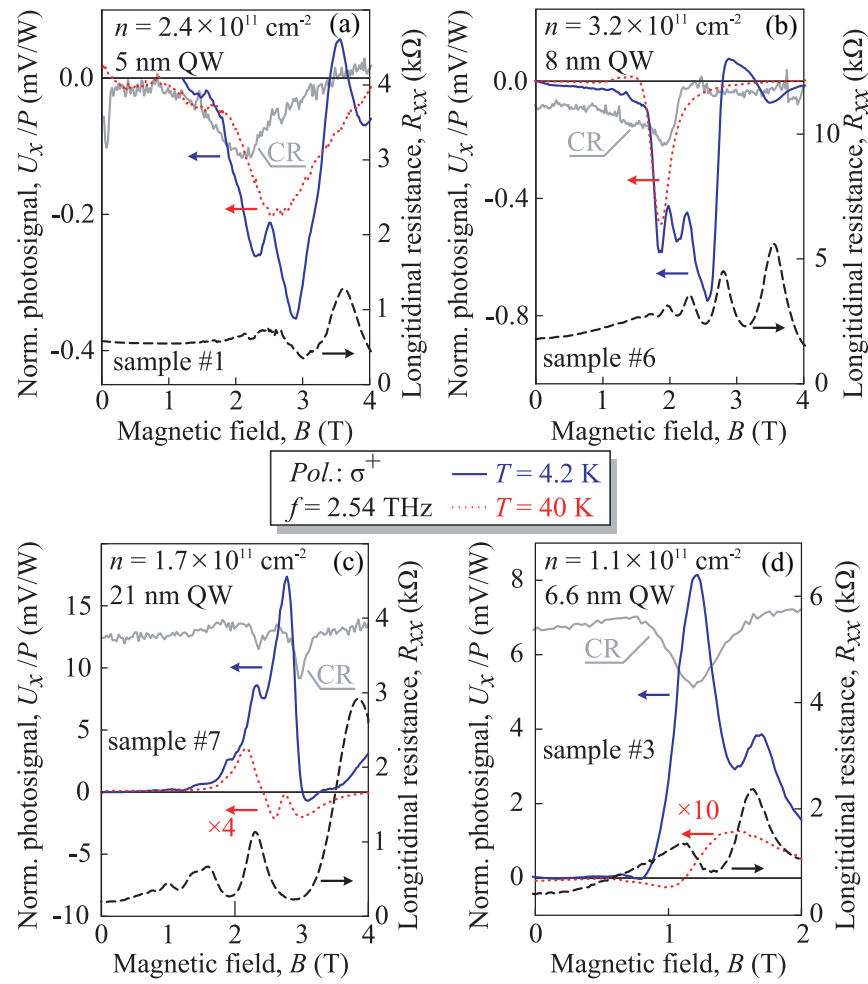

FIG. 5. (Color online) Photosignal normalized by radiation power $U_{x} / P$ (left scale) and $\mathrm{SdH}$ oscillations (right scale) for four different QW widths, (a) $L_{w}=5 \mathrm{~nm}$, (b) $L_{w}=8 \mathrm{~nm}$, (c) $L_{w}=21 \mathrm{~nm}$, and (d) $L_{w}=6.6 \mathrm{~nm}$ measured for two temperatures, $T=4.2 \mathrm{~K}$ (blue lines) and $T=40 \mathrm{~K}$ (dotted red lines). Grey lines indicated as "CR" show the change of the radiation transmission upon sweeping magnetic field measured at $T=4.2 \mathrm{~K}$. The data are given in arbitrary units.

$g$-factor absolute value [34]. As a result, for $\left|\Delta_{Z}\right|>\Delta E$ only odd numbers of minima can be detected. In fact, only odd minima in a certain magnetic field range have been detected in samples 1,5 , and 7 , which all have low electron density $n<2.5 \times 10^{11} \mathrm{~cm}^{-2}$. This is seen in magnetotransport data obtained in, e.g., 21-nm QWs for magnetic fields lower than $1.5 \mathrm{~T}$ as demonstrated in Fig. 5(c).

For the particular case of the linear dispersion, the cyclotron mass depends on the electron energy yielding nonequidistant Landau levels [1,41]. The $\mathrm{SdH}$ oscillations develop at the threshold field of $1.2 \mathrm{~T}$ which corresponds to the filling factor of 3; see Fig. 5(d) for sample 3 with 6.6-nm QW (van der Pauw geometry). With the further field increasing minima with filling factors 2 and 1 (not shown) are detected. The absence of the detectable higher filling factors in this sample is caused by the square-root dependence of the Landau level's energy being, as addressed above, characteristic for the Dirac fermion system. Higher filling factors up to 7, both odd and even, however, become visible in the carrier density dependence of the longitudinal resistance measured at a constant magnetic field in a gated 6.6-nm QW Hall bar sample 4; see Fig. 6. We note that the low-field regime where the broadening of energy levels exceeds by far the distance between them is not realized in all studied 6.6-nm samples. 


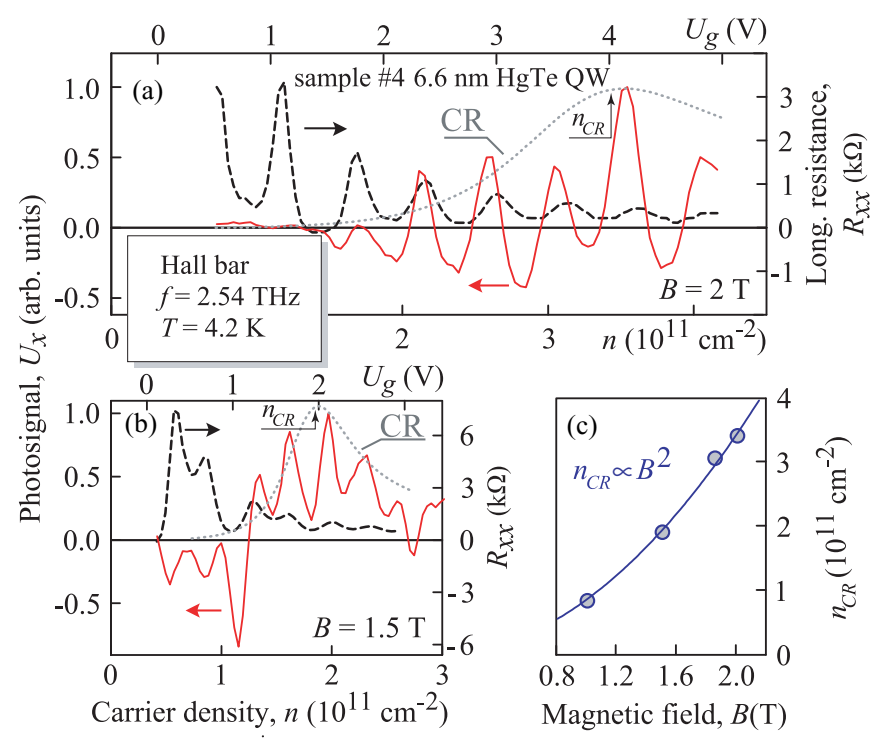

FIG. 6. (Color online) Normalized photosignals (left scale) and longitudinal resistance $R_{x x}$ (right scale) as functions of the electron density (bottom scale, for the corresponding gate voltage $U_{g}$ see top scale) measured for two different magnetic field values, $B=2 \mathrm{~T}$ [panel (a)] and 1.5 T [panel (b)]. Grey lines marked as "CR" in panels (a) and (b) show radiation absorption calculated after Eq. (21) for two values of magnetic field $B=2 \mathrm{~T}$ and $B=1.5 \mathrm{~T}$, respectively. Note that absorption is given in arbitrary units and all parameters used in the calculations are taken from experiments on magnetotransport and optical transmission performed for sample 4 . The velocity of Dirac fermions $v_{\mathrm{DF}}=7.6 \times 10^{5} \mathrm{~m} / \mathrm{s}$ for these calculations is taken close to that experimentally determined in Ref. [29] and $\tau_{\mathrm{tr}}=0.82 \mathrm{ps}$ at $n=10^{11} \mathrm{~cm}^{-2}$. (c) Magnetic field position of maximum photosignal as a function of electron density, showing a $n_{\mathrm{CR}} \propto B_{\mathrm{CR}}^{2}$ dependence. Solid lines in panels (a) and (b) show radiation absorption calculated after Eq. (21). Solid line in panel (c) shows $n_{\mathrm{CR}}$ vs $B$ dependence calculated after Eq. (2) applying the above parameters.

\section{Methods}

For optical excitation we apply a cw $\mathrm{CH}_{3} \mathrm{OH}$ laser emitting a radiation with frequency $f=2.54 \mathrm{THz}$ (wavelength $\lambda=$ $118.8 \mu \mathrm{m})$ and $f=1.62 \mathrm{THz}(\lambda=184 \mu \mathrm{m})[18,42]$. The incident radiation power $P \approx 10 \mathrm{~mW}$ is modulated at about $700 \mathrm{~Hz}$ by an optical chopper. The radiation at normal incidence is focused to a spot of about $1.5 \mathrm{~mm}$ diameter at the center of sample. The spatial beam distribution has an almost Gaussian profile which is measured by a pyroelectric camera [43]. Photocurrent, photoresistance, and optical transmission as functions of an applied magnetic field $\boldsymbol{B}$ have been studied, applying linearly as well as right- $\left(\sigma^{+}\right)$and left- $\left(\sigma^{-}\right)$handed circularly polarized radiation. The corresponding experimental setups are shown in Fig. 1. For electro-optical measurements the radiation induced electric current components $j_{x, y}$ have been measured via a voltage drop $U_{x, y} \propto j_{x, y}$, picked up across a $R_{i}=10 \mathrm{M} \Omega$ load resistor and applying the lock-in technique. While the photocurrent is measured in unbiased samples, to detect the photoresistance, we applied an external bias voltage $U_{B}$. Two methods have been used to obtain the change of the longitudinal resistance $\Delta R_{x x}(B)$. In the first one the photoresistive signal in response to the modulated radiation was determined by applying a dc voltage of $\pm 1 \mathrm{~V}$ to the sample. For a preresistor of $10 \mathrm{M} \Omega$ used in these experiments this bias voltage resulted in the current through the sample $I_{d c}=$ $\pm 0.1 \mu \mathrm{A}$. Both photoresistive and photocurrent responses have been extracted from the total photovoltage making use of the fact that the former changes its sign upon reversal of the bias polarity. Consequently, the half of the difference between the photosignals for positive and negative bias voltages allows one to obtain the photoresistance, whereas the half of their sum yields the bias voltage independent signal caused by the THzinduced photocurrent. In the second method, also providing the photoresistance $\Delta R_{x x}(B)$, we measure the longitudinal sample resistance by applying modulated $1-\mathrm{V}$ bias voltage (the corresponding current is about $0.1 \mu \mathrm{A}$ ) in the dark and under the illumination with nonmodulated $\mathrm{THz}$ radiation.

\section{EXPERIMENTAL RESULTS}

In (013)-oriented QWs excitation by normally incident $\mathrm{THz}$ radiation is known to result in photogalvanic $[20,44]$ and magnetophotogalvanic currents $[19,29]$. These photocurrents have already been studied in details being out of scope of the current paper. As addressed above here we focus on study in depth of the magnetic field-induced oscillations of the magnetophotogalvanic current observed at low temperatures under the conditions of the cyclotron resonance absorption. Note that the latter one has been widely studied in $\mathrm{HgTe}$ based materials [45-51].

\section{A. Results for parabolic dispersion}

We start with the data obtained for the 8- $\mathrm{nm} \mathrm{Hg}_{0.86} \mathrm{Cd}_{0.14} \mathrm{Te}$ QWs Hall bar sample 2 and representing QWs with normal parabolic bands order. Exciting the unbiased sample at zero magnetic field with linearly polarized radiation we detected the photocurrent exhibiting the characteristic behavior of the photogalvanic effect [20,44]. Sweeping the external magnetic field we observed that the photosignal shows the nonmonotonous behavior superimposed with oscillations and with a maximal signal at $B \approx 2.8 \mathrm{~T}$. The data for $T=4.2 \mathrm{~K}$ are plotted in Fig. 2(a). The observed oscillations correlate with $1 / B$-periodic oscillations of resistivity caused by the $\mathrm{SdH}$ effect, shown by black dashed lines in panels (a) and (b). The oscillatory behavior is also detected for the photoresistance signal, which, however, does not completely correlate with the SdH oscillations; see Fig. 2(b). Note that while the oscillations of photoresistance with similar features have been detected in HgTe-based and other low dimensional systems [52-55], the oscillations in the photocurrents generated in unbiased samples have not been observed so far.

We attribute the observed nonmonotonic behavior of the envelope of the photosignals, which is particularly clearly seen in the photoresistance data of Fig. 2(b), to the cyclotron resonance (CR). In order to verify this conjecture we switched to large area square-shaped samples, which allow us, in addition to the photoelectric experiments, to measure the radiation transmission. Studying the photocurrent and photoresistance in such samples we also observed oscillations. Figures $3-5$ show the photocurrent and photoresistance responses measured in pure $\mathrm{HgTe}$ QW samples of different QW widths characterized 
by normal ( $L_{w}=5 \mathrm{~nm}$, sample 1$)$ and inverted $\left(L_{w}=8 \mathrm{~nm}\right.$, samples 5 and 6, and $L_{w}=21 \mathrm{~nm}$, sample 7) band order. These figures also present the longitudinal resistance measured in the van der Pauw geometry and the radiation transmission. The photocurrent and photoresistance signals detected at liquid helium temperature in sample 5 with 8-nm QW, which is characterized by the almost parabolic dispersion with inverted band structure order, are shown in Figs. 3(a) and 4(b). Like in the data for sample 2 characterized by normal band order discussed above, see Fig. 2, the photocurrent detected in sample 5 exhibits multiple sign inversions and is enhanced in the vicinity of the cyclotron resonance detected by the radiation transmission; see Fig. 3(b). Similar behavior is detected in the photoresistance $\Delta R_{x x}$; see Fig. 4(b). Photoresistance data are obtained applying two methods described in Sec. IIC. In the first one $\Delta R_{x x}$ has been obtained applying dc bias voltage of either +1 or $-1 \mathrm{~V}$ and modulated radiation. In the second method we measured longitudinal resistance $R_{x x}$ in the dark and in the presence of unmodulated $\mathrm{THz}$ radiation applying standard magnetotransport setup, Fig. 4(a), and plotted the difference $\Delta R_{x x}$ in Fig. 4(c). The data reveal that, while illumination does not change the period of the $\mathrm{SdH}$ oscillations, it results in substantial decrease of their amplitude in the range of magnetic fields corresponding to the cyclotron resonance $\left(B_{\mathrm{CR}} \approx 2 \mathrm{~T}\right)$. Comparison of the photoresistance signal obtained by these two methods, see Fig. 4(c), shows that the results agree very well.

As the temperature increases the oscillations become less pronounced and almost vanish for $T=40 \mathrm{~K}$, so that both signals demonstrate a single resonance peak at $B_{\mathrm{CR}}=1.8 \mathrm{~T}$ for $f=2.54 \mathrm{THz}$ and $B_{\mathrm{CR}}=1.2 \mathrm{~T}$ for $f=1.64 \mathrm{THz}$. These data are shown for the photocurrent in Fig. 3(c). Clear resonances at the same magnetic field strength $B_{\mathrm{CR}}$ are also detected in the transmission experiments; see Fig. 3(b) for the radiation with $f=2.54 \mathrm{THz}$. Experiments applying right-handed circularly polarized radiation revealed that a resonance dip is present for the positive magnetic fields only. Switching the radiation helicity from $\sigma^{+}$to $\sigma^{-}$results in the resonance for negative magnetic fields ( $B_{\mathrm{CR}}=-1.8 \mathrm{~T}$ at $f=2.54 \mathrm{THz}$, not shown). For linearly polarized radiation being the superposition of $\sigma^{+}$ and $\sigma^{-}$photons, the resonance is observed for both magnetic field polarities. All these facts provide the evidence that the transmission dip at $B_{\mathrm{CR}}$, as well as the photosignal increase in the vicinity of $B_{\mathrm{CR}}$, are caused by the cyclotron resonance. From the cyclotron resonance position

$$
\left|B_{\mathrm{CR}}\right|=2 \pi f \frac{m_{c} c}{|e|}
$$

and its full width at half maximum, both determined from the radiation transmission data, we obtain effective mass $m_{c}=0.02 m_{0}$ and the scattering time $\tau_{\mathrm{CR}}=0.68 \mathrm{ps}$. Note that the latter value correlates well with the momentum scattering time $\tau_{\text {tr }}$, obtained from mobility; see Table I. Small deviations between these values detected in our experiments can be related with electron gas heating and radiative damping of the CR [56,57]. The same results are observed for other samples with inverted (samples 6 and 7) and noninverted (sample 1) parabolic dispersion; see for typical curves Figs. 5(a)-5(c). It is seen that all samples show an oscillatory behavior of the photocurrent at $T=4.2 \mathrm{~K}$ and a single peak at $T=40 \mathrm{~K}$; the latter corresponds to the cyclotron resonance position verified by the radiation transmission shown in the same figures.

\section{B. Results for a Dirac fermion system}

Now we turn to the measurements carried out on 6.6-nm QW samples 3 and 4, which are characterized by a linear energy spectrum [23,29]. In this system, carrier type and density have been controllably changed either by a gate voltage or optical illumination with red light-emitting diode (LED) in ungated samples (optical doping); see Table I where the illumination time $t_{\text {ill }}$ is indicated. The optical doping has been obtained using the persistent photoconductivity effect well known for $\mathrm{HgTe} / \mathrm{HgCdTe}$ QWs [23,25,29]. Figure 5(d) shows the magnetic field dependence of the photocurrent for sample 3. Similar to the data described above and obtained for the structures with almost parabolic dispersion, see Sec. III A, the photocurrent measured at $T=4.2 \mathrm{~K}$ exhibits oscillations correlating with the $\mathrm{SdH}$ oscillations, and the sample transmission has a clear cyclotron resonance dip at magnetic field $B=1.2 \mathrm{~T}$. Increasing the carrier density by the illumination with red LED we observed that the CR position, $B_{\mathrm{CR}}$, shifts to higher values by several times (not shown). The shift of the resonance caused by energy dependence of the cyclotron mass for the Dirac fermion systems and variation of the Fermi energy upon the illumination $[29,41]$ is described by (cf. Ref. [40]).

$$
\left|B_{\mathrm{CR}}\right|=\frac{(2 \pi)^{3 / 2} \sqrt{n} \hbar c f}{|e| v_{\mathrm{DF}}} .
$$

To obtain a fine tuning of the carrier density we performed additional measurements on the gated samples subjected to a constant magnetic field. The photocurrent together with the corresponding $\mathrm{SdH}$ oscillations detected in sample 4 is shown in Fig. 6 for two values of magnetic field. Besides the observed correlation between oscillations of the photocurrent and $\mathrm{SdH}$, the figure indicates the nonmonotonic behavior of the envelope function with the maximum at a density denoted as $n_{\mathrm{CR}}$. Performing these measurements for different values of the static magnetic field $B$, we observed that $n_{\mathrm{CR}}$ increases with rising magnetic field as $n_{\mathrm{CR}} \propto B^{2}$; see Fig. 6(c).

\section{THEORY}

The experiments discussed above demonstrate that photocurrent and photoresistance exhibit oscillations similar to the $\mathrm{SdH}$ oscillations of longitudinal resistivity. The oscillations, detected for all three types of electron dispersion, are enhanced at the cyclotron resonance and vanish with increasing the temperature, showing in this case only one peak in the signal being caused by the cyclotron resonance. In the following we present the theory describing the origin of the photogalvanic effect in classically strong magnetic fields where the electron cyclotron frequency $\omega_{c}$ exceeds the electron momentum scattering rate $1 / \tau_{\mathrm{tr}}$; this condition is certainly fulfilled for $B>0.5 \mathrm{~T}$ in all our samples. We show that the photocurrent oscillations, similarly to the de Haas-van Alphen and Shubnikov-de Haas effects, stem from the consecutive crossings of Fermi level by Landau levels. The peculiarities of quantum oscillations as functions of magnetic field and electron density are discussed. 


\section{A. Oscillations of photogalvanic current in quantizing magnetic field}

We begin with a model of the photocurrent generation developed on the basis of the spin-dependent asymmetric energy relaxation (relaxation mechanism [58,59]). In the framework of this model the Drude absorption of $\mathrm{THz}$ radiation leads to the electron gas heating [29]. The subsequent energy relaxation of the heated carriers in such materials becomes spin dependent, because the matrix element of electron scattering by phonons contains asymmetric spin-dependent terms $[58,60,61]$. Figure 7 sketches the cyclotron motion of electrons in the presence of asymmetric energy relaxation in two spin subbands $s_{z}= \pm 1 / 2$ in the case of the classically strong magnetic field, $\omega_{c} \tau_{\text {tr }} \gg 1$. We recall that $\omega_{c}=|e B| / m_{c} c$ is the cyclotron frequency, $c$ is the speed of light, $m_{c}$ is the cyclotron mass given by $m_{c}=\hbar^{2} k d k / d E, E=E(k)$ is the electron dispersion, and $\tau_{\mathrm{tr}}$ is the momentum (transport)
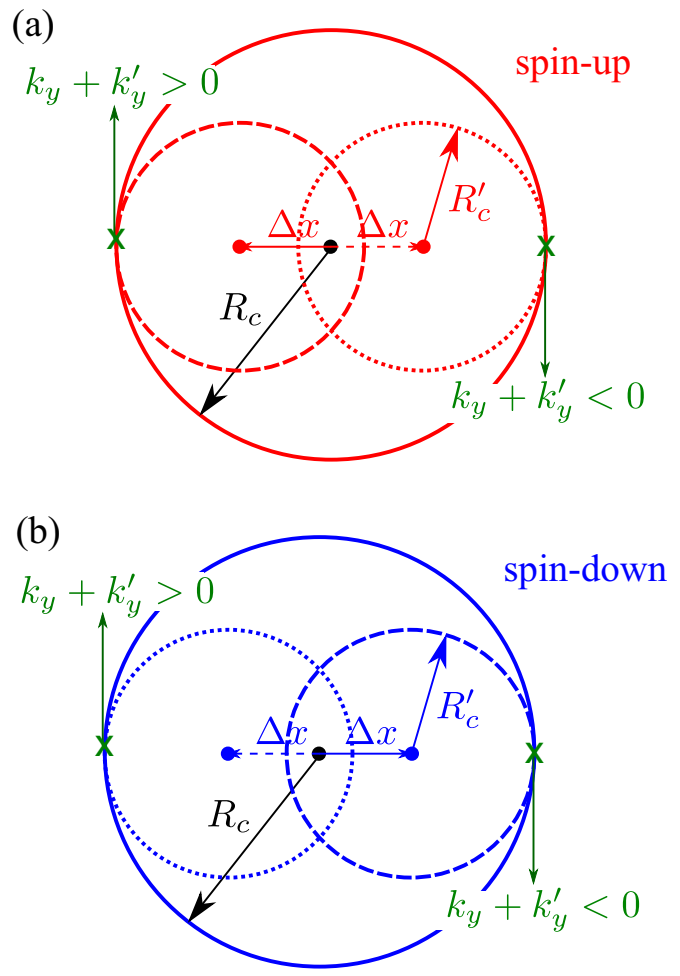

FIG. 7. (Color online) Mechanism of the photocurrent formation in the classically strong magnetic field, $\omega_{c} \tau_{\text {tr }} \gg 1$ in the spin-up subband (a) and in the spin-down subband (b). Solid circles depict the cyclotron orbit in the real space for the particle with given energy $E$. Cyclotron radius is $R_{c}$. Dashed and dotted red/blue circles depict cyclotron orbits for the electron after inelastic scattering by a phonon. Two possibilities for the scattering event are shown by crosses: at $k_{y}+k_{y}^{\prime}>0$ and at $k_{y}+k_{y}^{\prime}<0$. Due to change of the cyclotron radius, electron shifts in the real space by $\Delta x=\left|R_{c}-R_{c}^{\prime}\right|$, where $R_{c}^{\prime}$ is the cyclotron radius after the collision. The scattering processes are equally probable if wave-vector-dependent contribution $\propto\left(k_{y}+k_{y}^{\prime}\right)$ is neglected in the matrix element, Eq. (3). In this case any net shift of electron is absent. Allowance for the wave-vector-dependent contribution in Eq. (3) makes scattering with $k_{y}+k_{y}^{\prime}>0$ more probable giving rise to the flow in the spin-up subband directed along $x$ axis. scattering time. The classical cyclotron orbits of electrons in the spin-up subband are shown by closed circles of the cyclotron radius $R_{c}=v / \omega_{c}$, where $v \equiv v(k)=\hbar^{-1} \partial E / \partial k$ is the electron velocity. Without scattering the electron moves along the large circle shown by the solid line. We consider the dominating phonon-assisted relaxation process due to which the electron energy decreases. The scattering results in the displacement of the orbit center [62-64], and the orbits of smaller diameters are shown by dashed and dotted lines for two selected scattering points on the orbit with positive and negative values components of the wave vector $k_{y}$.

To take into account the spin-dependent scattering asymmetry we consider $\boldsymbol{k}$-linear terms in the electron-phonon interaction matrix elements for spins aligned along the magnetic field $\boldsymbol{B}$, which as we show below, are relevant for the photocurrent generation [65]. The corresponding matrix element has the form

$$
V_{\boldsymbol{k}^{\prime} \boldsymbol{k}}=V_{0}+V_{1} \sigma_{z}\left(k_{y}+k_{y}^{\prime}\right),
$$

where the first term in the right-hand side describes the conventional spin-independent scattering, $\sigma_{z}$ is the Pauli matrix, $\boldsymbol{k}$ and $\boldsymbol{k}^{\prime}$ are the initial and final wave vectors. We emphasize that the above terms are allowed for gyrotropic media only $[58,66,67]$ and have been considered for HgTe-based quantum wells with both parabolic [19] and linear dispersions [29]. Evidently, the shift of orbits for positive and negative $k_{y}$ are opposite, resulting in the shifts of electrons parallel or antiparallel to the $x$ axis. For the fixed spin, e.g., spin-up in Fig. 7(a), spin dependent scattering makes the probabilities of these events unequal [higher for $k_{y}>0$ and lower for $k_{y}<0$; see Eq. (3)], which results in the steady electron flow along the $x$ axis, $i_{x,+}$. For the opposite spin, see Fig. 7(b), the situation reverses and the flow $i_{x,-}$ is oppositely directed. Consequently, in the absence of Zeeman effect we obtain a pure spin current. However, the magnetic field induced Zeeman splitting causing unequal electron subband populations and mobilities in each spin subband makes the magnitudes of the flows unequal giving rise to the net dc current, $j_{x}=e\left(i_{x,+}+i_{x,-}\right)$.

Quantum effects enter the picture as an interference of electron waves on classical orbits yielding Landau levels. As is well known, the quantization results in $1 / B$-periodic oscillations of density of states and of scattering rates caused by the crossing of the Fermi level $E_{F}$ by Landau levels $[63,64,68]$. In particular, we show that oscillations of the photocurrent stem from periodic variation of the radiation absorption rates, occupations of spin-up and spin-down subbands $n_{ \pm}$, and electron scattering rates $W_{\boldsymbol{k}^{\prime} \boldsymbol{k}}^{ \pm}$.

Formally, the electron fluxes in the $x$ direction are given by the product of elementary displacement of the charge carrier in the real space due to the scattering event and its probability,

$$
i_{x, \pm}=\sum_{\boldsymbol{k}, \boldsymbol{k}^{\prime}}\left(x_{\boldsymbol{k}}-x_{\boldsymbol{k}^{\prime}}\right) W_{\boldsymbol{k}^{\prime} \boldsymbol{k}}^{ \pm}
$$

where the position of the cyclotron orbit center is given by

$$
x_{k}=\hbar k_{x} \frac{c}{|e B|} .
$$

These expressions are general and valid for parabolic as well as for linear dispersions [69]. Considering the scattering on phonons and assuming that at low temperatures the 
thermalization between the spin branches due to electronelectron collisions is not efficient [70], we obtain the following expression for the total dc current:

$$
j_{x}=e\left(i_{x,+}+i_{x,-}\right)=\frac{e \beta \zeta c}{|B|}\left|E_{0}\right|^{2}\left[n_{+} \mu_{+}(\omega)-n_{-} \mu_{-}(\omega)\right],
$$

where $E_{0}$ is the complex amplitude of the incident radiation, $\beta=1$ for parabolic and $\beta=1 / 2$ for linear energy dispersion, $\mu_{ \pm}(\omega)$ are the high-frequency electron mobilities in each spin branch related with the high-frequency (ac) dissipative conductivities as $\sigma_{ \pm}(\omega)=|e| n_{ \pm} \mu_{ \pm}(\omega)$, and

$$
\zeta=\frac{2 \operatorname{Re}\left(V_{0} V_{1}^{*}\right)}{\left|V_{0}\right|^{2}} \frac{k}{v(k)}
$$

is the small parameter responsible for the scattering asymmetry [74]. It follows from Eq. (6) that the photocurrent is proportional to the squared amplitude of the electromagnetic field, i.e., to the radiation intensity, as well as to the radiation absorption rate in the corresponding spin subband $\propto n_{ \pm} \mu_{ \pm}(\omega)$. We stress that Eqs. (4)-(6) hold in both classical and quantizing magnetic fields provided that $\hbar \omega_{c} \ll E_{F}$, where $E_{F}$ is the Fermi energy, if $\boldsymbol{k}, \boldsymbol{k}^{\prime}$ are replaced by the appropriate quantum numbers in the magnetic field, namely, $N$ (the Landau-level number) and $P_{x}=\hbar k_{x}-e A_{x} / c$ the generalized momentum ( $A_{x}=-B y$ is the vector potential of the static field), and all quantities are expressed via $N, N^{\prime}, P_{x}, P_{x}^{\prime}$ [75]. The quantum oscillations of the photocurrent $\boldsymbol{j}$, Eq. (6), originate from the oscillations of $n_{ \pm}$and $\mu_{ \pm}$.

It is convenient to decompose the current $j$ as a sum of two components: one, $j_{n}$, related to the spin polarization $S_{z}=\left(n_{+}-n_{-}\right) / 2 n$ in the system (de Haas-van Alphen effect) and the other, $j_{\mu}$, related solely with the difference, $\mu_{+}(\omega)-\mu_{-}(\omega)$, of high-frequency mobilities in spin subbands (Shubnikov-de Haas effect); total electron density $n=n_{+}+n_{-}$. To do this we rewrite the term in square brackets in Eq. (6) in the form

$$
S_{z} n\left[\mu_{+}(\omega)+\mu_{-}(\omega)\right]+n \frac{\mu_{+}(\omega)-\mu_{-}(\omega)}{2} .
$$

Then

$$
j_{x}=j_{n}+j_{\mu},
$$

where

$$
\begin{gathered}
j_{n}=\frac{2 e \zeta c \beta\left|E_{0}\right|^{2}}{|B|} S_{z} n \mu(\omega), \\
j_{\mu}=\frac{e \zeta c \beta\left|E_{0}\right|^{2}}{|B|} n \frac{\mu_{+}(\omega)-\mu_{-}(\omega)}{2},
\end{gathered}
$$

and $\mu(\omega)=\left[\mu_{+}(\omega)+\mu_{-}(\omega)\right] / 2$. The above equations show that the oscillatory part of the photocurrent is, in fact, contained in the magnetic field dependence of (i) the electronspin polarization $S_{z}$ (contribution $j_{n}$ ), and (ii) the mobility difference in subbands with opposite spins, $\mu_{+}(\omega)-\mu_{-}(\omega)$ (contribution $j_{\mu}$ ). Thus, below we focus on these quantities and derive the expressions for them in the cases of QWs with parabolic and linear energy dispersions.

\section{B. Photocurrent in the system with parabolic dispersion}

To be specific we first analyze the case of parabolic dispersion characterized by the energy-independent effective mass $m$, and, in line with experiment, the frequency range of $\omega$ in the vicinity of the cyclotron frequency $\omega_{c}$.

We start with calculation of electron spin $S_{z}$ determining the first contribution to the photocurrent $j_{n}$. With allowance for the quantum oscillations (de Haas-van Alphen effect), it is given by (cf. Ref. [76])

$$
S_{z}=\frac{1}{2} \frac{\int_{0}^{E_{F}}\left[v_{+}(E)-v_{-}(E)\right] d E}{\int_{0}^{E_{F}}\left[v_{+}(E)+v_{-}(E)\right] d E},
$$

where $v_{ \pm}(E)=v_{0}\left(E \mp \Delta_{Z} / 2\right)$ are the densities of states in each spin branch with $\nu_{0}(E)$ being the density of states per spin found neglecting Zeeman effect. Here $\Delta_{Z}=g \mu_{B} B$ is the Zeeman splitting with $g$ being the electron Landé factor and $\mu_{B}$ being Bohr magneton. We consider classical magnetic fields, where $\hbar \omega_{c} \ll E_{F}$ and for $E_{F} \tau_{q} / \hbar \gg 1$. Here $\tau_{q}$ is the quantum scattering time related to Dingle temperature, which describes the lifetime of an electron in a given quantum state, and is shorter than the transport time $\tau_{\text {tr }}$ for a smooth disorder potential. Under these assumptions the density of states can be written as $[64,77,78]$

$$
\nu_{0}(E)=\frac{m}{2 \pi \hbar^{2}}\left[1-2 \exp \left(-\frac{\pi}{\omega_{c} \tau_{q}}\right) \cos \left(2 \pi \frac{E}{\hbar \omega_{c}}\right)\right],
$$

where we have taken into account the oscillating contributions of the first order in small parameter $\exp \left(-\pi / \omega_{c} \tau_{q}\right)$ describing the interference of electron waves on the cyclotron orbits [63]. The spin polarization is proportional to the energy-integrated difference of densities of states in spin-up and spin-down subbands, Eq. (10), namely,

$$
\begin{aligned}
S_{z}= & -\frac{1}{4 E_{F}}\left[\Delta_{Z}-\frac{2 \hbar \omega_{c}}{\pi} \mathcal{T}_{1} \sin \left(\frac{\pi \Delta_{Z}}{\hbar \omega_{c}}\right)\right. \\
& \left.\times \cos \left(\frac{2 \pi E_{F}}{\hbar \omega_{c}}\right) e^{-\pi / \omega_{c} \tau_{q}}\right] .
\end{aligned}
$$

Note that hereinafter we neglect the oscillatory corrections of higher orders in small parameters $\hbar \omega_{c} / E_{F} \ll 1$ and $\Delta_{Z} / E_{F} \ll 1$. The factor

$$
\mathcal{T}_{1}=\frac{2 \pi^{2} k_{B} T_{e}}{\hbar \omega_{c} \sinh \left(2 \pi^{2} k_{B} T_{e} / \hbar \omega_{c}\right)}
$$

takes into account a thermal spread of the electron distribution function with $T_{e}$ being the electron-gas temperature. Interestingly, the oscillatory contribution to the spin polarization $S_{z}$ being proportional to the factor $\sin \left(\pi \Delta_{Z} / \hbar \omega_{c}\right)$ is absent if Zeeman splitting $\Delta_{Z}$ is a multiple of the inter-Landau-level distance $\hbar \omega_{c}$. In this case the Landau levels in both spin branches are aligned [79] and oscillations of spin polarization vanish.

Now we turn to the high-frequency mobilities in each spin subband whose difference gives rise to $j_{\mu}$. In line with Refs. $[64,80,81]$ we have for $\omega$ in the vicinity of $\omega_{c}$

$$
\begin{aligned}
\mu_{ \pm}(\omega)= & \frac{|e| \tau_{\mathrm{tr}} / 2 m}{1+\left(\omega-\omega_{c}\right)^{2} \tau_{\mathrm{tr}}^{2}}\left[1+\mathcal{T}_{1} e^{-\pi / \omega_{c} \tau_{q}} \cos \left(\frac{2 \pi E_{F_{ \pm}}}{\hbar \omega_{c}}\right)\right. \\
& \left.\times F\left(\omega \tau_{\mathrm{tr}}, \omega_{c} \tau_{\mathrm{tr}}\right)\right]
\end{aligned}
$$


with $E_{F_{ \pm}}=E_{F} \mp \Delta_{Z} / 2$ and

$$
\begin{aligned}
F\left(\omega \tau_{\mathrm{tr}}, \omega_{c} \tau_{\mathrm{tr}}\right)= & \frac{2\left(\omega-\omega_{c}\right)^{2} \tau_{\mathrm{tr}}^{2}}{1+\left(\omega-\omega_{c}\right)^{2} \tau_{\mathrm{tr}}^{2}} \frac{\sin 2 \pi \omega / \omega_{c}}{2 \pi \omega / \omega_{c}} \\
& +\frac{1+3\left(\omega-\omega_{c}\right)^{2} \tau_{\mathrm{tr}}^{2}}{1+\left(\omega-\omega_{c}\right)^{2} \tau_{\mathrm{tr}}^{2}} \frac{\sin ^{2} \pi \omega / \omega_{c}}{\left(\omega-\omega_{c}\right) \tau_{\mathrm{tr}} \pi \omega / \omega_{c}}
\end{aligned}
$$

is the smooth (on the scale of $\hbar \omega_{c} / E_{F}$ ) function of $\omega \tau_{\mathrm{tr}}$ and $\omega_{c} \tau_{\text {tr }}$. Here we neglect a background contribution to the mobility resonant at $\omega=-\omega_{c}$ [82]. Equation (14) shows that the high-frequency mobilities contain both the classical (Drude) CR part, being proportional to $\tau_{\mathrm{tr}} /\left[1+\left(\omega-\omega_{c}\right)^{2} \tau_{\mathrm{tr}}^{2}\right]$, and $1 / B$-periodic oscillatory contributions resulting from the consecutive crossing of Fermi level by Landau levels $[64,80,83]$.

Taking into account Eqs. (10) and (14) we can obtain from Eqs. (8) and (9) contributions $j_{n}$ and $j_{\mu}$ in the form

$$
\begin{aligned}
j_{n}= & -\frac{e \zeta c\left|E_{0}\right|^{2} n \mu(\omega)}{2 E_{F}|B|}\left[\Delta_{Z}-\frac{2 \hbar \omega_{c}}{\pi} \mathcal{T}_{1} e^{-\pi / \omega_{c} \tau_{q}} \sin \left(\frac{\pi \Delta_{Z}}{\hbar \omega_{c}}\right)\right. \\
& \left.\times \cos \left(\frac{2 \pi E_{F}}{\hbar \omega_{c}}\right)\right],
\end{aligned}
$$

and

$$
\begin{aligned}
j_{\mu}= & \frac{e^{3} \zeta c\left|E_{0}\right|^{2} n}{2 m|e B|} \frac{\tau_{\mathrm{tr}}}{1+\left(\omega-\omega_{c}\right)^{2} \tau_{\mathrm{tr}}^{2}} \mathcal{T}_{1} e^{-\pi / \omega_{c} \tau_{q}} \sin \left(\frac{\pi \Delta_{Z}}{\hbar \omega_{c}}\right) \\
& \times \sin \left(\frac{2 \pi E_{F}}{\hbar \omega_{c}}\right) F\left(\omega \tau_{\mathrm{tr}}, \omega_{c} \tau_{\mathrm{tr}}\right)
\end{aligned}
$$

respectively. Equations (16) and (17) describe the classical smooth part and quantum oscillations of the photocurrent in systems with parabolic dispersion.

\section{Photocurrent in the system with linear dispersion}

In the HgTe-based quantum wells of critical thickness the electron energy spectrum at zero magnetic field is linear, $E(k)=\hbar v_{\mathrm{DF}} k$. Our theoretical treatment demonstrates that the basic mechanism of the photocurrent generation is the same as in the HgTe-based QWs with normal or inverted parabolic band structure. The photocurrent contains two contributions $j_{n}$ and $j_{\mu}$ resulting from the different populations of spin subbands and from the difference in high-frequency mobilities in these subbands and described by Eqs. (8) and (9), respectively. In the case of linear dispersion, however, the Landau levels are not equidistant and the quantum oscillation pattern changes. Here we present the set of formulas which generalize Eqs. (11)-(14) to the case of linear dispersion taking into account, as in Sec. IV B, only first-order oscillatory contributions. The density of states assumes the form [40] (see also
Ref. [84])

$$
v_{0}(E)=\frac{m_{c}}{2 \pi \hbar^{2}}\left[1+2 \exp \left(-\frac{\pi}{\omega_{c} \tau_{q}}\right) \cos \left(\frac{\pi E}{\hbar \omega_{c}}\right)\right],
$$

where both cyclotron mass $m_{c} \equiv m_{c}(E)=E / v_{\mathrm{DF}}^{2}$ and cyclotron frequency $\omega_{c} \equiv \omega_{c}(E)=e B v_{\mathrm{DF}}^{2} /(E c)$ are the functions of electron energy. Under the same approximations as for the parabolic spectrum we obtain the electron-spin polarization in the first order in $\exp \left(-\pi / \omega_{c} \tau_{q}\right)$ :

$$
\begin{aligned}
S_{z}= & -\frac{1}{2 E_{F}}\left[\Delta_{Z}+\frac{2 \hbar \omega_{c}}{\pi} \mathcal{T}_{1} e^{-\pi / \omega_{c} \tau_{q}} \sin \left(\frac{\pi \Delta_{Z}}{\hbar \omega_{c}}\right)\right. \\
& \left.\times \cos \left(\frac{\pi E_{F}}{\hbar \omega_{c}}\right)\right],
\end{aligned}
$$

and the high-frequency mobilities in spin-up and spin-down branches,

$$
\begin{aligned}
\mu_{ \pm}(\omega)= & \frac{e \tau_{\mathrm{tr}} / 2 m_{c}}{1+\left(\omega-\omega_{c}\right)^{2} \tau_{\mathrm{tr}}^{2}}\left[1-\mathcal{T}_{1} e^{-\pi / \omega_{c} \tau_{q}} \cos \left(\frac{\pi E_{F_{ \pm}}}{\hbar \omega_{c}}\right)\right. \\
& \left.\times F\left(\omega \tau_{\mathrm{tr}}, \omega_{c} \tau_{\mathrm{tr}}\right)\right]
\end{aligned}
$$

where the function $F\left(\omega \tau_{\mathrm{tr}}, \omega_{c} \tau_{\mathrm{tr}}\right)$ is given by Eq. (15) [85]. In Eqs. (19) and (20) one has to put $E=E_{F}$ in $m_{c}, \omega_{c}, \tau_{q}$, and $\tau_{\text {tr. }}$. Note that in the case of linear dispersion even short-range scattering results in different quantum (out-scattering) time $\tau_{q}$ and transport time $\tau_{\mathrm{tr}}=2 \tau_{q}$. Equations (19) and (20) allow us to calculate the photocurrent contributions $j_{n}$ and $j_{\mu}$ by Eqs. (8) and (9) for the system with linear energy dispersion.

\section{DISCUSSION}

The theory discussed in the previous section allows us to describe the experimental data. First we discuss the basic features of the photocurrent in the quantum wells with parabolic dispersion, then we address peculiarities of the quantum wells of critical thickness, particularly, the appearance of the cyclotron resonance in the photocurrent as a function of the carrier density. The photoresistivity effect is discussed in the end of this section as well.

\section{A. Quantum oscillations of photocurrent in QWs with parabolic dispersion}

To analyze the oscillations of the photocurrent generated in the structures with almost parabolic dispersion [86] we calculated the individual contributions $j_{n}$ and $j_{\mu}$, as well as the total photocurrent $j=j_{n}+j_{\mu}$ using Eqs. (16), (17), and Eq. (7), respectively. Figure 8 shows the results obtained using an effective mass $m=0.034 m_{0}$, carrier density $n=7.5 \times 10^{11} \mathrm{~cm}^{-2}$, and the electron $g$ factor $g=-36.5$, i.e., the parameters close to that of sample 2 with $8-\mathrm{nm} \mathrm{QW}$. Note that large values of electron $g$ factors in HgTe-based QWs were reported in Ref. [87]. Comparison of the total photocurrent plotted in Fig. 8(c) with experimental result for sample 2 representing QWs with the normal band order [Fig. 2(a)] clearly shows that the main experimental features are fully reproduced by the theoretical calculations. First of all, both calculated and experimental signals show pronounced 


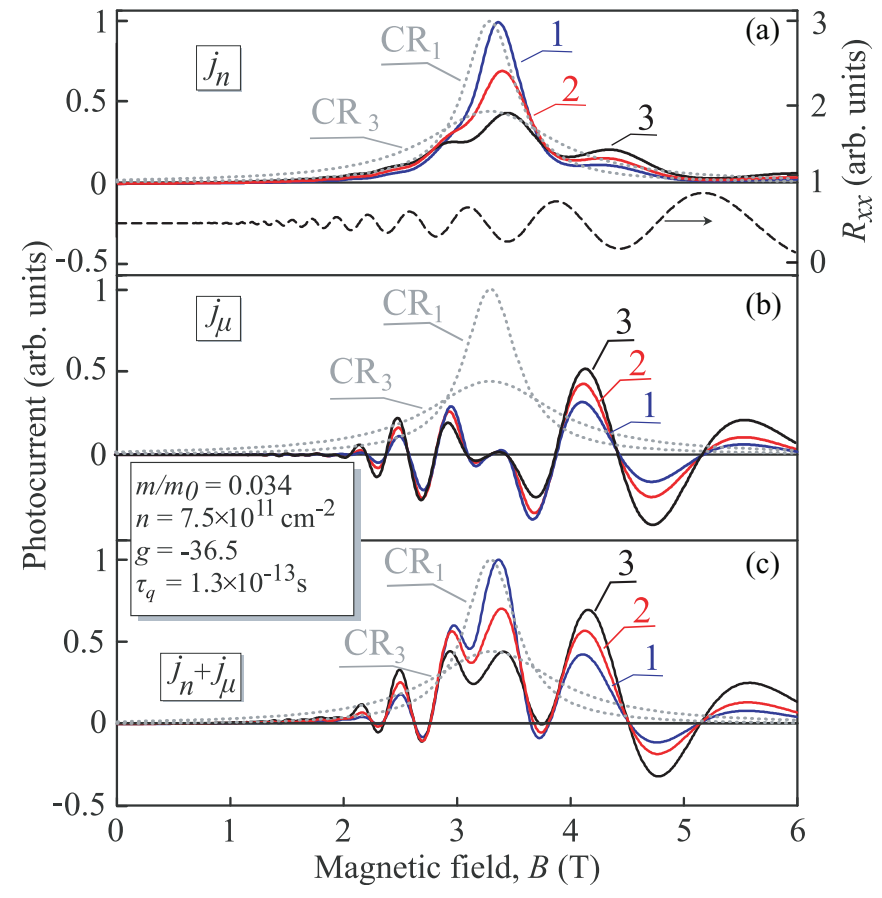

FIG. 8. (Color) Panels (a) and (b) show photocurrent contributions $j_{n}$ and $j_{\mu}$ calculated for different transport times $\tau_{t r}$ after Eqs. (16) and (17), respectively. The total photocurrent given by the sum of $j_{n}$ and $j_{\mu}$, see Eq. (7), is presented in panel (c). Curves $1-3$ are calculated for $\tau_{\mathrm{tr}}=7.4 \times 10^{-13}, 5 \times 10^{-13}$, and $3 \times 10^{-13} \mathrm{sec}$, respectively. The parameters used in the calculations are chosen close to those of sample $2: m=0.034 m_{0}, n=7.5 \times 10^{11} \mathrm{~cm}^{-2}, g=-36.5$, and $\tau_{q}=1.3 \times 10^{-13} \mathrm{sec}$. The normalization is the same for all panels. Dashed lines show calculated cyclotron resonance absorption profiles $\mathrm{CR}_{1}$ and $\mathrm{CR}_{3}$ obtained for $\tau_{\mathrm{tr}}=7.4 \times 10^{-13}$ and $3 \times 10^{-13} \mathrm{sec}$, respectively. These data are given in arbitrary units with the $\mathrm{CR}$ maximum almost equal to $j_{n}$ maximum. Dashed line in (a) shows the first harmonic in the oscillatory part of $R_{x x}$.

oscillations accompanied with inversion of the current direction. They start to be observable in the range of magnetic fields where the cyclotron resonance takes place [see the dashed line in Fig. 8(a)]. Moreover, both experiment and theory reveal that the oscillations for magnetic fields $B>\left|B_{\mathrm{CR}}\right|$ are substantial being comparable with that in the vicinity of the cyclotron resonance. Different magnetic field behavior of the photocurrent caused by spin polarization, $j_{n}$, and that driven by the difference of mobilities in the spin branches, $j_{\mu}$, provides a way to distinguish their contributions to the total photocurrent. Figures 8(a) and 8(b) show that, while $j_{n}$ almost follows the cyclotron resonance, being slightly modulated by the periodic sign-conserved oscillations $j_{\mu}$, in contrast, is characterized by the multiple reversal of the current direction with maxima far away from CR. Moreover, the latter contribution vanishes at CR position. Figure 9 shows that while $j_{n}$ contribution to the photocurrent achieves maximum at $\mathrm{CR}$ position and rapidly decreases outside the resonance, the oscillations of this term are almost absent. By contrast, the contribution $j_{\mu}$ vanishes exactly in the resonance and shows oscillations, with one period of oscillations being pronounced beyond the resonance, $B>B_{\mathrm{CR}}$. Experimental data shown in Fig. 2(a) reveal that $j_{n}$ and $j_{\mu}$ components of the photocurrent are comparable.

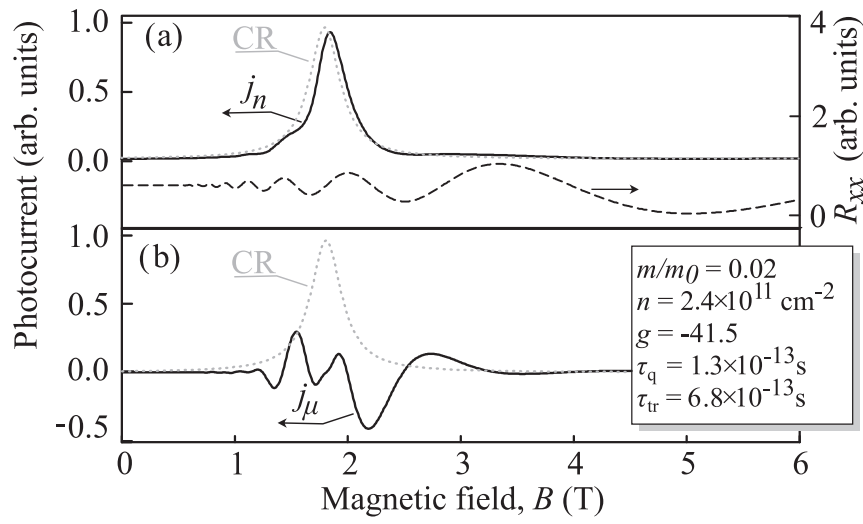

FIG. 9. Panels (a) and (b) show photocurrent contributions $j_{n}$ and $j_{\mu}$ calculated after Eqs. (16) and (17), respectively. Dotted lines show calculated cyclotron resonance absorption. Photocurrent contributions and $\mathrm{CR}$ profile are normalized to their maximum values. Dashed line in (a) shows first-order oscillatory contribution to $R_{x x}$. The parameters used in the calculations are chosen close to those of sample 5: $m=0.02 m_{0}, n=2.4 \times 10^{11} \mathrm{~cm}^{-2}, g=-41.5$, $\tau_{q}=1.3 \times 10^{-13} \mathrm{sec}, \tau_{\mathrm{tr}}=6.8 \times 10^{-13} \mathrm{sec}$.

In particular, the multiple sign inversion of the photocurrent, being the fingerprint of $j_{\mu}$ is clearly detected demonstrating that this mechanism dominates in the total current. The same results are obtained for sample 5 representing structures with the inverted band order, see Fig. 3(a), being in agreement with the corresponding calculations, see Fig. 9.

While Figs. 8 and 9 present the calculations for a relatively broad cyclotron resonance, Fig. 10 shows the results of calculation for relatively narrow $\mathrm{CR}$ which covers only one period of $\mathrm{SdH}$ oscillations, see dotted and dashed lines in Fig. 10 for $\mathrm{CR}$ and $\mathrm{SdH}$ oscillations, respectively. This condition is relevant to the low temperature measurements in QWs with normal $\left(L_{w}=5 \mathrm{~nm}\right)$ and inverted $\left(L_{w}=8\right.$ and $21 \mathrm{~nm})$ parabolic bands summarized in Figs. 5(a)-5(c). The

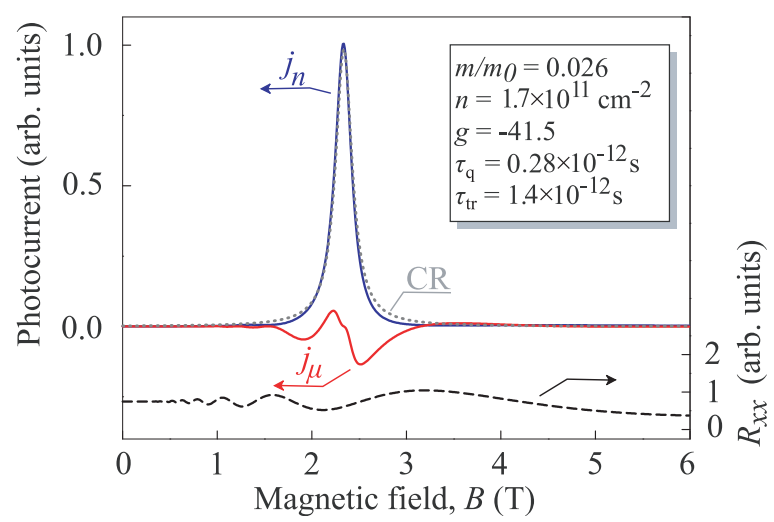

FIG. 10. (Color online) Photocurrent contributions $j_{n}$ and $j_{\mu}$ calculated after Eqs. (16) and (17), respectively. Dotted line shows calculated cyclotron resonance absorption. Absorption and $j_{n}$ contribution to the photocurrent are normalized to their maximum values. Dashed line shows first-order oscillatory contribution to $R_{x x}$. The parameters used in the calculations are chosen close to those of sample $7: m=0.026 m_{0}, n=1.7 \times 10^{11} \mathrm{~cm}^{-2}, g=-41.5$, $\tau_{q}=0.28 \times 10^{-12} \mathrm{sec}, \tau_{\mathrm{tr}}=1.4 \times 10^{-12} \mathrm{sec}$. 
parameters used in the calculations are chosen close to those of sample \#7. The results of the calculations describe well the experimental findings, see Fig. 5(c) [88]. Comparison of the calculated $j_{n}$ and $j_{\mu}$ with experimental data shown in Figs. 5(a)-5(c) demonstrates that while both contributions can be clearly identified the main input in these structures comes from $j_{n}$. This conclusion is proved by the higher signals at $\mathrm{CR}$ position as well as by a small number of the detected oscillations [90].

Below we analyze in more detail a complex picture of the photocurrent oscillations. We start with the photocurrent $j_{n}$, which is proportional to the electron-spin polarization $S_{z}$, given by Eq. (16) and plotted in Figs. 8(a) and 9(a); see also blue solid curve in Fig. 10. This contribution to the photocurrent represents the dc current generated due to a difference of spin-up and spin-down subbands populations in the presence of magnetic field, see Eq. (8). The effect is also known as a zero-bias spin separation [58] converted to the electric current due to Zeeman effect, which has been observed in many semiconductor low-dimensional systems; for review see Refs. [37,67,94,95]. One can see that the dominating input comes from a smooth nonoscillatory part which almost follows the cyclotron resonance and is in agreement with Eqs. (13) and (14) of Ref. [29]. However, since both $S_{z}$ and $\mu(\omega)$ in Eq. (8) contain also $1 / B$-periodic components, the $j_{n}$ contains relatively small [for $\exp \left(-\pi / \omega_{c} \tau_{q}\right) \ll 1$ ] quantum oscillations described by $\cos \left(2 \pi E_{F} / \hbar \omega_{c}\right)$ superimposed over a smooth background, as demonstrated in Fig. 8(a). Similarly to de Haas-van Alphen and Shubnikov-de Haas effects scattering processes and thermal spread of electron distribution function suppress the oscillations of $S_{z}$ and $\mu_{ \pm}$; see Eqs. (12) and (14). Therefore, only the smooth part of the $j_{n}$ contribution remains responsible for the observed resonant photocurrent at high temperatures $T \simeq 40 \mathrm{~K}$, where oscillations vanish; see Figs. 3(b) and 5.

Now we turn to the second contribution $j_{\mu}$, which results from the magnetic field induced difference of high-frequency mobilities in spin-up and spin-down branches; see Eqs. (9) and (14). It is given by Eq. (17) and plotted in Figs. 8 and Fig. 9(b) as well as by the red solid curve in Fig. 10. As seen from the figure this contribution also oscillates as a function of magnetic field, but compared to $j_{n}$ does not have any smooth part and demonstrates multiple sign inversions. The latter is due to the fact that the difference of mobilities $\mu_{ \pm}(\omega)$ mainly comes from oscillatory factors $\sin \left(2 \pi E_{F} / \hbar \omega_{c}\right)$ [96]. In other words, the direction of the $j_{\mu}$ current is determined by the electron flux in the spin branch with extremal density of states at the Fermi level. As magnetic field changes, either the spin-up or spin-down branch dominates, and the photocurrent changes its direction. The amplitude of the oscillations of $j_{\mu}$ comes from two competing factors: an increase of Shubnikov-de Haas oscillations amplitude with an increase of magnetic field and the decrease of radiation absorption for $B>B_{\mathrm{CR}}$. Moreover, $j_{\mu}$ cancels at exact resonance position, $B=B_{\mathrm{CR}}$, and, depending on the relation between the scattering times $\tau_{q}$ and $\tau_{\mathrm{tr}}$ and on the value of $\omega_{c} \tau_{\mathrm{tr}}$ the photocurrent $j_{\mu}$ can either show oscillations for $B>B_{\mathrm{CR}}$ or be suppressed. Formally, the oscillations for $B>B_{\mathrm{CR}}$ stem from an increase of quantum parameter $\exp \left(-\pi / \omega_{c} \tau_{q}\right)$, which governs the amplitude of the magneto-oscillations in Eqs. (16) and (17), resulting also in the increase of Shubnikov-de Haas oscillations amplitude in the resistivity of the QW structure.

Theoretical Fig. 8 as well as experimental Figs. 2(a) and 3(a) show that $j_{n}$ and $j_{\mu}$ contributions have at low temperatures close magnitudes. It stems from the fact that $j_{n}$ contains small parameter $\Delta_{Z} / E_{F}$ since this term is proportional to the spin polarization $S_{z}$. By contrast, $j_{\mu}$ contains the small quantum parameter, namely, $\exp \left(-\pi / \omega_{c} \tau_{q}\right)$, while Zeeman splitting $\Delta_{Z}$ enters only as a ratio $\Delta_{Z} / \hbar \omega_{c}$, which can be on the order of unity. For typical parameters $\Delta_{Z} / \hbar \omega_{c} \sim 1, \exp \left(-\pi / \omega_{c} \tau_{q}\right)=$ $0.1 \ldots 0.5$ the contributions are comparable.

It is noteworthy that there is a phase shift between oscillatory parts of $j_{n}$ and $j_{\mu}$ contributions to the photocurrent. Particularly, as it follows from Eq. (16), the oscillations of $j_{n}$ have the same phase as or inverted phase in respect to the Shubnikov-de Haas oscillations of resistivity given by the factor $\cos \left(2 \pi E_{F} / \hbar \omega_{c}\right)$. By contrast, according to Eq. (17), the oscillations of $j_{\mu}$ are given by the factor $\sin \left(2 \pi E_{F} / \hbar \omega_{c}\right)$ and are phase shifted by $\pi / 2$ in respect to the Shubnikov-de Haas oscillations.

The total photocurrent is given by the sum of $j_{n}$ and $j_{\mu}$ contributions and demonstrates the rather complex oscillatory behavior shown in Fig. 8(c). Particularly, the oscillations are accompanied with sign inversion, but they are superimposed over a smooth CR-like background. An interplay of $j_{n}$ and $j_{\mu}$ contributions may result in the overall phase shift of the photocurrent oscillations in respect to the Shubnikov-de Haas oscillations of the sample resistivity, like that observed, e.g., for 8-nm QW; see Fig. 3(a). It is worthwhile to stress that in addition to the above considered photocurrent $j=$ $j_{n}+j_{\mu}$ resulting from the spin-dependent asymmetric energy relaxation mechanism the measured photocurrent may contain contributions caused by the spin dependent asymmetry of optical transitions (excitation mechanism; see Ref. [58]). The latter mechanism also results in the magneto-oscillations, and the functional form of its individual contributions is similar to that of $j_{n}$ and $j_{\mu}$. Hence, for detailed comparison of experimental data and theory one needs to take into account these additional photocurrent contributions as well as higher-order terms in Eqs. (16) and (17) in quantum parameter $\exp \left(-\pi / \omega_{c} \tau_{q}\right)$, as experimental data presented in Figs. 2 and 4 reveal second harmonic in Shubnikov-de Haas oscillations of resistivity; see also Sec. V C.

\section{B. Quantum oscillations of photocurrent in QWs with linear dispersion}

Now we turn to the system with Dirac dispersion realized in quantum wells of critical thickness, $L_{w}=6.6 \mathrm{~nm}$ (samples 3 and 4). The analysis performed in Sec. IV C shows that the magnetic field dependence of the photocurrent is similar to that in the system with normal or inverted parabolic band. This result is in agreement with the experimental data presented in Fig. 5(d). Particular differences, cf. different panels of Fig. 5, are mostly quantitative due to different values of electron densities, scattering times, effective masses, etc. Indeed, the comparison of Eqs. (19) and (12) as well as Eqs. (20) and (14) for the photocurrent contributions in the systems with linear and parabolic dispersions, respectively, shows that the differences between them are the amplitude and the phase of 
quantum oscillations. The former one is primary determined by strong dependence of the cyclotron mass on the electron energy in QWs of critical thickness which is almost absent in systems with parabolic dispersion. Similarly to the systems with parabolic dispersion, a phase shift between the photocurrent and Shubnikov-de Haas oscillations comes from the interplay of $j_{n}$ and $j_{\mu}$ addressed above, as observed in experiment; see Fig. 6(a).

A distinguishing feature of the photocurrent in the Dirac fermions system, however, is the fact that the CR-resonance related effects change upon the carrier density variation. It becomes possible due to the linear energy spectrum resulting in the dependence of the cyclotron frequency on the electron Fermi energy $E_{\mathrm{F}} \propto \sqrt{n}$. The $\mathrm{CR}$ resonance as a function of the carrier density or gate voltage can be understood by considering the electron density dependence of the highfrequency conductivity. The latter determines the radiation absorption and, in the vicinity of the cyclotron resonance, can be recast as

$$
\sigma(\omega)=\frac{e^{2} n^{*} \tau_{\mathrm{tr}}^{*}}{2 m_{c}^{*}\left[1+\left(\omega-\omega_{c}^{*} \eta\right)^{2} \tau_{\mathrm{tr}}^{* 2} \eta^{2}\right]},
$$

where parameters $m_{c}^{*}, \tau_{\mathrm{tr}}^{*}$, and $\omega_{c}^{*}$ denote corresponding quantities at some (arbitrary) electron density $n=n^{*}$ and $\eta=\sqrt{n^{*} / n}$. Equation (21) implies short-range scattering. The effect of electron density variation is demonstrated in Figs. 11 and 12 where the photocurrent is plotted as a function of electron density for two values of magnetic field, $B=2$ and $1.5 \mathrm{~T}$, respectively. Note that all parameters used in the calculations are taken from experiments on magnetotransport and optical transmission performed for sample 4. One can

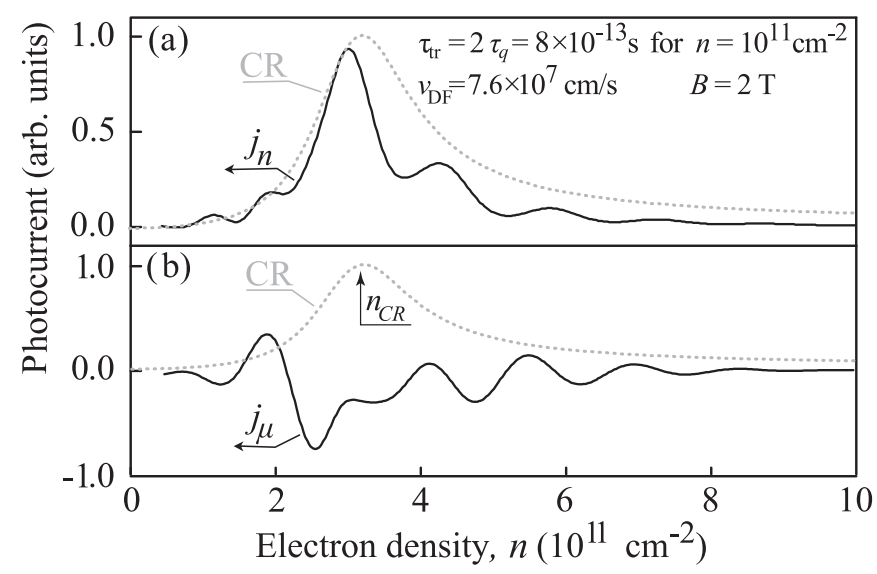

FIG. 11. Panels (a) and (b) show photocurrent contributions $j_{n}$ and $j_{\mu}$ calculated after Eqs. (8) and (9), respectively. Spin polarization and the high-frequency mobilities in the opposite spin branches are calculated for $B=2 \mathrm{~T}$ after (19) and (20) applying velocity of Dirac fermions $v_{\mathrm{DF}}=7.6 \times 10^{7} \mathrm{~cm} / \mathrm{s}$ close to that experimentally determined in Ref. [29]. In the calculations we assume the shortrange scattering with $\tau_{q}=\tau_{\mathrm{tr}} / 2=4 \times 10^{-13} \mathrm{sec}$ for electron density $n=10^{11} \mathrm{~cm}^{-2}$. Dotted lines show CR profile as a function of electron density. The radiation absorption is calculated after Eq. (21) for $B=2 \mathrm{~T}$. All parameters used in the calculations are taken from experiments on magnetotransport and optical transmission performed for sample 4. Note that the photocurrent contributions and CR profile are normalized to their maximum values.

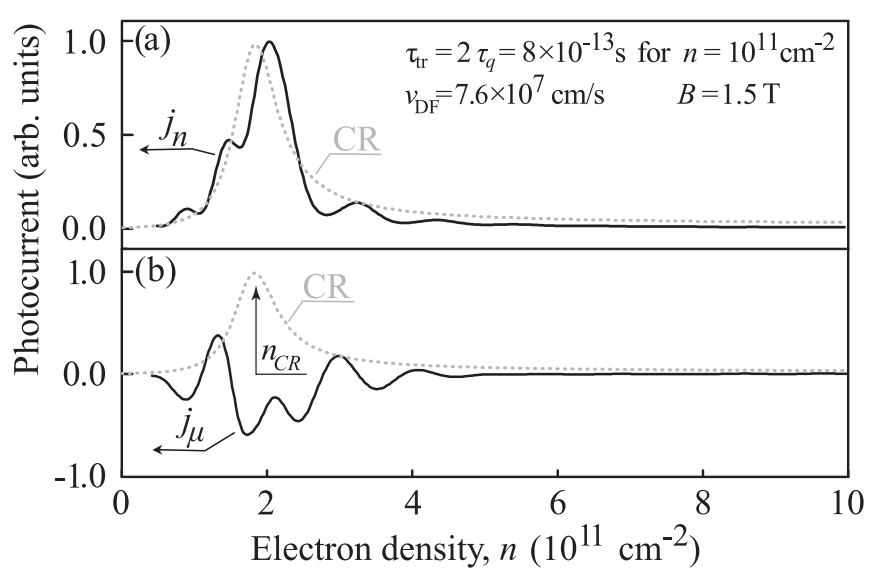

FIG. 12. Panels (a) and (b) show photocurrent contributions $j_{n}$ and $j_{\mu}$ calculated after Eqs. (8) and (9), respectively. Spin polarization and the high-frequency mobilities in the opposite spin branches are calculated for $B=1.5 \mathrm{~T}$ after (19) and (20) applying velocity of Dirac fermions $v_{\mathrm{DF}}=7.6 \times 10^{7} \mathrm{~cm} / \mathrm{s}$ close to that experimentally determined in Ref. [29]. In the calculations we assume the shortrange scattering with $\tau_{q}=\tau_{\text {tr }} / 2=4 \times 10^{-13} \mathrm{sec}$ for electron density $n=10^{11} \mathrm{~cm}^{-2}$. Dotted lines show CR profile as a function of electron density. The radiation absorption is calculated after Eq. (21) for $B=1.5 \mathrm{~T}$. All parameters used in the calculations are taken from experiments on magnetotransport and optical transmission performed for sample 4. Note that the photocurrent contributions and CR profile are normalized to their maximum values.

see that the photocurrent as a function of electron density shows the pronounced resonance superimposed with quantum oscillations. Interestingly, for the linear electron dispersion $j_{\mu}$ contribution related with the difference of spin-up and spin-down subbands mobilities contains a smooth background caused by the dependence of the cyclotron mass and scattering times on electron energy. Both figures show that the resonance position is close to the CR peak denoted as $n_{\mathrm{CR}}$ (see dotted lines) and shifts towards the smaller densities with a decrease of the $B$ field. This is in agreement with experimental data for sample 4 presented in Fig. 6 together with the absorption calculated after Eq. (21) (see grey full lines). The dependence of $n_{\mathrm{CR}}$ on magnetic field calculated after Eq. (21) agrees well with that obtained in the experiment; see Fig. 6(c). Interestingly, Figs. 6(a) and 6(b) show that while the $\mathrm{SdH}$ oscillations in the longitudinal resistance decrease and almost vanish with raising carrier density, the amplitude of the photocurrent oscillations gets strongly increased. This highlights the fact that the photocurrent is enhanced at the cyclotron resonance position, which in sample 4 corresponds to rather high carrier density.

To summarize this part, the photocurrent in unbiased HgTebased QWs in the presence of magnetic field demonstrates $1 / B$-periodic oscillations. The developed theory based on the spin-dependent asymmetric energy relaxation shows that the photocurrent oscillations stem from the crossing of Fermi level by Landau levels. The analysis shows that the photocurrent contains two contributions $j_{n}$ and $j_{\mu}$ caused by the electronspin polarization in the magnetic field and by the difference of spin-up and spin-down subband mobilities. 


\section{Photoresistance (photoconductivity) effect}

Finally, for completeness, we address the oscillations of the photoresistance and compare these results with the experiment. For the analysis of the photoresistance $\Delta R_{x x}$ we will use the data for 8-nm QW sample 5 shown in Fig. 4(c). The microscopic origin of the photoresistance is related with the electron-gas heating caused by the absorption of $\mathrm{THz}$ radiation and a corresponding reduction of the electron mobility; for review see, e.g., [35]. In the model where the electron gas is described by an effective temperature $T_{e}$ the heating process is governed by the energy balance equation:

$$
\frac{k_{B}\left(T_{e}-T_{l}\right)}{\tau_{\epsilon}}=\frac{2|e|}{n}\left[\mu_{+}(\omega) n_{+}+\mu_{-}(\omega) n_{-}\right]\left|E_{0}\right|^{2},
$$

where $T_{l}$ is the lattice temperature and $\tau_{\epsilon}$ is the energy relaxation time. The photoconductance tensor is, by definition, given by

$$
\sigma_{\alpha \beta}^{(\mathrm{ph})}=\Delta\left(\sigma_{\alpha \beta}\right) \equiv \sigma_{\alpha \beta}\left(T=T_{e}\right)-\sigma_{\alpha \beta}\left(T=T_{l}\right),
$$

where $\sigma_{\alpha \beta}$ is the static conductivity, and the photoresistance signal measured experimentally is given by

$$
\Delta R_{x x} \propto \Delta\left(\frac{\sigma_{x x}}{\sigma_{x x}^{2}+\sigma_{x y}^{2}}\right)
$$

$\Delta(.$.$) denotes the variation of some quantity under illumina-$ tion. We recall that, similarly to Eq. (14), the components of static conductivity also contain oscillatory contributions. Particularly, for quantum wells with parabolic electron dispersion,

$$
R_{x x} \propto 1+2 \mathcal{T}_{1} \exp \left(-\frac{\pi}{\omega_{c} \tau_{q}}\right) \cos \left(\pi \frac{\Delta_{Z}}{\hbar \omega_{c}}\right) \cos \left(2 \pi \frac{E_{F}}{\hbar \omega_{c}}\right) .
$$

Hence, an increase of an electron-gas effective temperature $T_{e}$ mainly results in the suppression of the oscillatory factor because coefficient $\mathcal{T}_{1}$ given by Eq. (13) exponentially decreases with an increase of temperature. As a result, the pronounced oscillatory photoresistance appears. The envelope function of the photoresistance oscillations at $B \lesssim B_{\mathrm{CR}}$ approximately follows the cyclotron resonance line shape, because the heating is most efficient exactly in the resonance and becomes weaker the larger detuning, $\left|\omega_{c}-\omega\right|$. However, the oscillations of the photoresistance signal do not decay as fast as cyclotron resonance for $B>\left|B_{\mathrm{CR}}\right|$ because the oscillatory contributions increase with an increase of magnetic field. This is illustrated in Fig. 13 where panel (a) shows the oscillatory part of resistivity calculated for the parameters close to that of $L_{w}=8-\mathrm{nm}$ sample 5, while panel (b) represents the photoresistance signal. In contrast to the calculations of photocurrents presented above, here we took into account both first and second harmonics of SdH oscillations, see below. The calculated photoresistance signal is presented in Fig. 4(c) (blue solid curve) together with the measured photoresistance (red solid curve) and calculated $\mathrm{CR}$ radiation absorption (dashed curve). Apart a difference in the amplitudes the calculations are in a reasonable agreement with experiment.

At last but not at least we address the influence of the ratio between the Landau-level separation and Zeeman splitting. In linear transport for $\Delta_{Z} /\left(\hbar \omega_{c}\right) \approx 1 / 2$ the first harmonic in

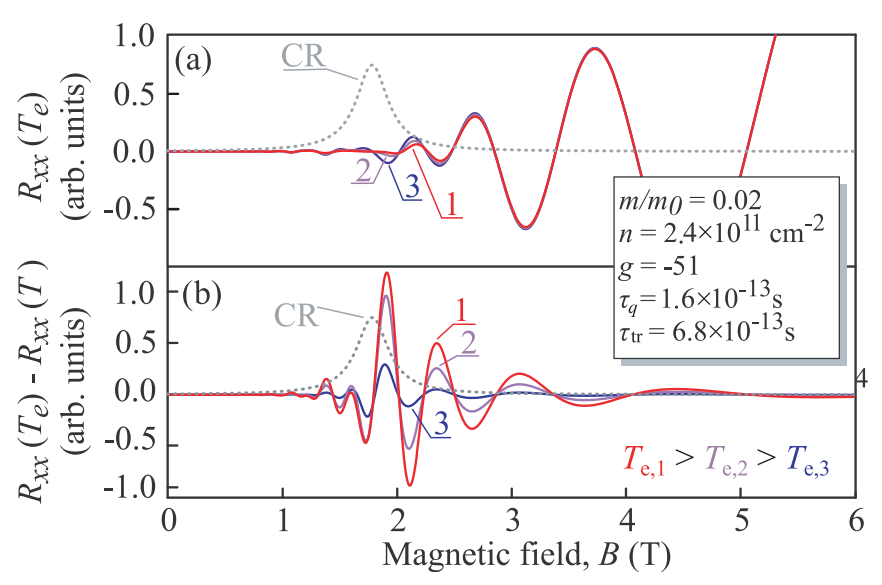

FIG. 13. (Color) Oscillatory part of the resistivity (a) and the photoresistive signal (b) calculated after Eqs. (23)-(26), respectively. Curves 1, 2, and 3 correspond to different electron temperatures, $T_{e}$, being $\propto \tau_{\epsilon}\left|E_{0}\right|^{2}$; see Eq. (22). The parameters are chosen close to those of 8-nm sample 5: $m=0.02 m_{0}, n=2.4 \times 10^{11} \mathrm{~cm}^{-2}, g=-51$, $\tau_{q}=1.6 \times 10^{-13} \mathrm{sec}$, momentum relaxation time $\tau_{\mathrm{tr}}=6.8 \times 10^{-13} \mathrm{sec}$. Both first- and second-order oscillatory contributions are taken into account and additional phase shift $\varphi=-\pi / 5$ was included to match the phase of experimental data in Fig. 4. Dotted lines show calculated cyclotron resonance absorption. The data are given in arbitrary units.

quantum oscillations of static resistivity vanishes; see Eq. (25) and Ref. [79] for details. This is caused by the mismatch of Landau levels and spin sublevels. Hence, second harmonic in the resistivity oscillations described by

$$
\mathcal{T}_{2} \exp \left(-\frac{2 \pi}{\omega_{c} \tau_{q}}\right) \cos \left(2 \pi \frac{\Delta_{Z}}{\hbar \omega_{c}}\right) \cos \left(4 \pi \frac{E_{F}}{\hbar \omega_{c}}\right)
$$

with $\mathcal{T}_{2}=4 \pi^{2} k_{B} T_{e}\left[\hbar \omega_{c} \sinh \left(4 \pi^{2} k_{B} T_{e} / \hbar \omega_{c}\right)\right]^{-1}$ becomes important. It results also in the frequency doubling of the photoresistance signal as clearly seen in Fig. 13. This effect is essential to describe the experimental data both on photoresistance and on $\mathrm{SdH}$ oscillations. By contrast, for the previously discussed photocurrent and the spin-polarization oscillations both the first and second harmonic vanish simultaneously if $\Delta_{Z} /\left(\hbar \omega_{c}\right) \approx 1$. It is because photocurrent is caused by the imbalance of electron fluxes in two spin branches, therefore its extrema are realized when the mismatch of the Zeeman and Landau levels is maximal.

To conclude this section we note that at high lattice temperatures where oscillatory corrections to the conductivity governed by factors $\mathcal{T}_{1}$ and $\mathcal{T}_{2}$ vanish, the photoresistance can be still caused by the electron-gas heating and corresponding change of the classical conductivity, i.e., via temperature dependence of the scattering times. In this case the photoresistance signal as a function of magnetic field follows the Lorentzian shape of cyclotron resonance depicted by dotted curves in Fig. 13 in agreement with experimental results, Fig. 3(c).

\section{CONCLUSIONS}

To conclude, the detailed experimental and theoretical studies of quantum magneto-oscillations of photocurrent in 
HgTe-based quantum wells have been performed. It has been demonstrated that $1 / B$-periodic oscillations stem from the consecutive crossing of Fermi level by Landau levels and they become strongly enhanced at the cyclotron resonance conditions. The oscillations have been observed as a function of magnetic field for all three types of electron dispersion realized in HgTe quantum wells: normal parabolic, inverted parabolic, and linear. Similar responses detected in all investigated samples evidence the universality of the effect. The developed theory explains photocurrent formation as a result of the spin-dependent asymmetric energy relaxation. It demonstrates that the photocurrent contains two contributions resulting from (i) the magnetic field induced electron spin polarization and (ii) the difference of the electron mobilities in the spin-up and spin-down subbands in the presence of magnetic field, both of which contain oscillatory component. The theory describes well all main experimental features.

\section{ACKNOWLEDGMENTS}

Authors thank B. McCombe and S. A. Tarasenko for discussions. The support from the DFG (SPP 1666), Elite Network of Bavaria (K-NW-2013-247), RFBR, RAS, and Russian President Grants No. NSh-5062.2014.2 and No. NSh-1085.2014.2 is gratefully acknowledged.
[1] A. H. Castro Neto, F. Guinea, N. M. R. Peres, K. S. Novoselov, and A. K. Geim, Rev. Mod. Phys. 81, 109 (2009).

[2] K. S. Novoselov, V. I. Fal'ko, L. Colombo, P. R. Gellert, M. G. Schwab, and K. Kim, Nature (London) 490, 192 (2012).

[3] M. M. Glazov and S. D. Ganichev, Phys. Rep. 535, 101 (2014).

[4] M. Z. Hasan and C. L. Kane, Rev. Mod. Phys. 82, 3045 (2010).

[5] J. E. Moore, Nature (London) 464, 194 (2010).

[6] X.-L. Qi, S. C. Zhang, and X. L. Qi, Rev. Mod. Phys. 83, 1057 (2011).

[7] B. A. Bernevig, T. L. Hughes, and S. C. Zhang, Science 314, 1757 (2006).

[8] M. S. König, S. Wiedmann, C. Brüne, A. Roth, H. Buhmann, L. Molenkamp, X. L. Qi, and S. C. Zhang, Science 318, 766 (2007).

[9] A. Roth, C. Brüne, H. Buhmann, L. W. Molenkamp, J. Maciejko, X.-L. Qi, and S.-C. Zhang, Science 325, 294 (2009).

[10] K. C. Nowack, E. M. Spantor, M. Baenninger, M. König, J. R. Kirtley, B. Kalisky, C. Ames, P. Leubner, C. Brüne, H. Buhmann, L. W. Molenkamp, D. Goldhaber-Gordon, and K. A. Moler, Nat. Mater. 12, 787 (2013).

[11] Z. D. Kvon, K.-M. Dantscher, C. Zoth, D. A. Kozlov, N. N. Mikhailov, S. A. Dvoretsky, and S. D. Ganichev, JETP Lett. 99, 290 (2014).

[12] L. Fu and C. L. Kane, Phys. Rev. B 76, 045302 (2007).

[13] X. Dai, T. L. Hughes, X.-L. Qi, Z. Fang, and S.-C. Zhang, Phys. Rev. B 77, 125319 (2008).

[14] C. Brüne, C. X. Liu, E. G. Novik, E. M. Hankiewicz, H. Buhmann, Y. L. Chen, X. L. Qi, Z. X. Shen, S. C. Zhang, and L. W. Molenkamp, Phys. Rev. Lett. 106, 126803 (2011).

[15] J. B. Oostinga, L. Maier, P. Schüffelgen, D. Knott, C. Ames, C. Brüne, G. Tkachov, H. Buhmann, and L. W. Molenkamp, Phys. Rev. X 3, 021007 (2013).

[16] D. A. Kozlov, Z. D. Kvon, E. B. Olshanetsky, N. N. Mikhailov, S. A. Dvoretsky, and D. Weiss, Phys. Rev. Lett. 112, 196801 (2014).

[17] M. I. Dyakonov and A. V. Khaetskii, JETP Lett. 33, 110 (1981).

[18] Z. D. Kvon, S. N. Danilov, N. N. Mikhailov, S. A. Dvoretski, and S. D. Ganichev, Physica E 40, 1885 (2008).

[19] H. Diehl, V. A. Shalygin, L. E. Golub, S. A. Tarasenko, S. N. Danilov, V. V. Bel'kov, E. G. Novik, H. Buhmann, C. Brüne, L. W. Molenkamp, E. L. Ivchenko, and S. D. Ganichev, Phys. Rev. B 80, 075311 (2009).
[20] B. Wittmann, S. N. Danilov, V. V. Bel'kov, S. A. Tarasenko, E. G. Novik, H. Buhmann, C. Brüne, L. W. Molenkamp, E. L. Ivchenko, Z. D. Kvon, N. N. Mikhailov, S. A. Dvoretsky, N. Q. Vinh, A. F. G. van der Meer, B. Murdin, and S. D. Ganichev, Semicond. Sci. Technol. 25, 095005 (2010).

[21] A. V. Ikonnikov, A. A. Lastovkin, K. E. Spirin, M. S. Zholudev, V. V. Rumyantsev, K. V. Maremyanin, A. V. Antonov, V. Ya. Aleshkin, V. I. Gavrilenko, S. A. Dvoretskii, N. N. Mikhailov, Yu. G. Sadofyev, and N. Samal, JETP Lett. 92, 756 (2010).

[22] B. Büttner, C. X. Liu, G. Tkachov, E. G. Novik, C. Brüne, H. Buhmann, E. M. Hankiewicz, P. Recher, B. Trauzettel, S. C. Zhang, and L. W. Molenkamp, Nat. Phys. 7, 418 (2011).

[23] Z. D. Kvon, S. N. Danilov, D. A. Kozlov, C. Zoth, N. N. Mikhailov, S. A. Dvoretskii, and S. D. Ganichev, JETP Lett. 94, 816 (2011).

[24] J. N. Hancock, J. L. M. van Mechelen, A. B. Kuzmenko, D. van der Marel, C. Brüne, E. G. Novik, G. V. Astakhov, H. Buhmann, and L. W. Molenkamp, Phys. Rev. Lett. 107, 136803 (2011).

[25] A. V. Ikonnikov, M. S. Zholudev, K. E. Spirin, A. A. Lastovkin, K. V. Maremyanin, V. Ya. Aleshkin, V. I. Gavrilenko, O. Drachenko, M. Helm, J. Wosnitza, M. Goiran, N. N. Mikhailov, S. A. Dvoretskii, F. Teppe, N. Diakonova, C. Consejo, B. Chenaud, and W. Knap, Semicond. Sci. Technol. 26, 125011 (2011).

[26] M. S. Zholudev, A. V. Ikonnikov, F. Teppe, M. Orlita, K. V. Maremyanin, K. E. Spirin, V. I. Gavrilenko, W. Knap, S. A. Dvoretskiy, and N. N. Mihailov, Nanoscale Res. Lett. 7, 534 (2012).

[27] A. V. Ikonnikov, M. S. Zholudev, K. V. Maremyanin, K. E. Spirin, A. A. Lastovkin, V. I. Gavrilenko, S. A. Dvoretskii, and N. N. Mikhailov, JETP Lett. 95, 406 (2012).

[28] D. A. Kozlov, Z. D. Kvon, N. N. Mikhailov, and S. A. Dvoretsky, JETP Lett. 96, 730 (2012).

[29] P. Olbrich, C. Zoth, P. Vierling, K.-M. Dantscher, G. V. Budkin, S. A. Tarasenko, V. V. Bel'kov, D. A. Kozlov, Z. D. Kvon, N. N. Mikhailov, S. A. Dvoretsky, and S. D. Ganichev, Phys. Rev. B 87, 235439 (2013).

[30] A. M. Shuvaev, G. V. Astakhov, G. Tkachov, C. Brüne, H. Buhmann, L. W. Molenkamp, and A. Pimenov, Phys. Rev. B 87, 121104 (2013).

[31] A. Shuvaev, A. Pimenov, G. V. Astakhov, M. Mühlbauer, C. Brüne, H. Buhmann, and L. W. Molenkamp, Appl. Phys. Lett. 102, 241902 (2013). 
[32] M. Orlita, D. M. Basko, M. S. Zholudev, F. Teppe, W. Knap, V. I. Gavrilenko, N. N. Mikhailov, S. A. Dvoretskii, P. Neugebauer, C. Faugeras, A.-L. Barra, G. Martinez, and M. Potemski, Nat. Phys. 10, 233 (2014).

[33] S. D. Ganichev, E. L. Ivchenko, and W. Prettl, Physica E 14, 166 (2002).

[34] E. L. Ivchenko, Optical Spectroscopy of Semiconductor Nanostructures (Alpha Science, Harrow, UK, 2005).

[35] S. D. Ganichev and W. Prettl, Intense Terahertz Excitation of Semiconductors (Oxford University Press, New York, 2006).

[36] E. L. Ivchenko and S. D. Ganichev, in Spin Photogalvanics in Spin Physics in Semiconductors, edited by M. I. Dyakonov (Springer, New York, 2008).

[37] V. V. Bel'kov and S. D. Ganichev, Semicond. Sci. Technol. 23, 114003 (2008).

[38] Z. D. Kvon, E. B. Olshanetsky, N. N. Mikhailov, and D. A. Kozlov, Low Temp. Phys. 35, 6 (2009).

[39] S. Das Sarma, Shaffique Adam, E. H. Hwang, and Enrico Rossi, Rev. Mod. Phys. 83, 407 (2011).

[40] U. Briskot, I. A. Dmitriev, and A. D. Mirlin, Phys. Rev. B 87, 195432 (2013).

[41] S. Masubuchi, M. Onuki, M. Arai, T. Yamaguchi, K. Watanabe, T. Taniguchi, and T. Machida, Phys. Rev. B 88, 121402(R) (2013).

[42] J. Karch, P. Olbrich, M. Schmalzbauer, C. Zoth, C. Brinsteiner, M. Fehrenbacher, U. Wurstbauer, M. M. Glazov, S. A. Tarasenko, E. L. Ivchenko, D. Weiss, J. Eroms, R. Yakimova, S. Lara-Avila, S. Kubatkin, and S. D. Ganichev, Phys. Rev. Lett. 105, 227402 (2010).

[43] E. Ziemann, S. D. Ganichev, I. N. Yassievich, V. I. Perel, and W. Prettl, J. Appl. Phys. 87, 3843 (2000).

[44] S. N. Danilov, B. Wittmann, P. Olbrich, W. Eder, W. Prettl, L. E. Golub, E. V. Beregulin, Z. D. Kvon, N. N. Mikhailov, S. A. Dvoretsky, V. A. Shalygin, N. Q. Vinh, A. F. G. van der Meer, B. Murdin, and S. D. Ganichev, J. Appl. Phys. 105, 013106 (2009).

[45] J. R. Meyer, C. A. Hoffman, F. J. Bartoli, T. Wojtowicz, M. Dobrowolska, J. K. Furdyna, X. Chu, J. P. Faurie, and L. R. Ram-Mohan, J. Vac. Sci. Technol. B10, 1582 (1992).

[46] M. Schultz, U. Merkt, A. Sonntag, U. Rössler, T. Colin, P. Helgesen, T. Skauli, and S. Lovold, J. Cryst. Growth 184/185, 1180 (1998).

[47] M. Schultz, U. Merkt, A. Sonntag, U. Rössler, R. Winkler, T. Colin, P. Helgesen, T. Skauli, and S. Lovold, Phys. Rev. B 57, 14772 (1998).

[48] M. Schultz, F. Heinrichs, U. Merkt, T. Colin, T. Skauli, and S. Løvold, Semicond. Sci. Technol. 11, 1168 (1996).

[49] M. von Truchsess, V. Latussek, C. R. Becker, and E. Batke, J. Cryst. Growth 159, 1104 (1996).

[50] V. J. Goldman, H. D. Drew, M. Shayegan, and D. A. Nelson, Phys. Rev. Lett. 56, 968 (1986).

[51] C.-M. Hu, C. Zehnder, C. Heyn, and D. Heitmann, Phys. Rev. B 67, 201302 (2003).

[52] V. G. Golubev, V. I. Ivanov-Omskii, and G. I. Kropotov, Sov. Tech. Phys. Lett. 3, 216 (1977).

[53] Z. D. Kvon, D. A. Kozlov, S. N. Danilov, C. Zoth, P. Vierling, S. Stachel, V. V. Bel'kov, A. K. Bakarov, D. V. Dmitriev, A. I. Toropov, and S. D. Ganichev, JETP Lett. 97, 41 (2013).

[54] M. Pakmehr, A. Stier, B. D. McCombe, C. Brüne, H. Buhmann, and L. Molenkamp, Proc. of EP2DS-20, ThOE13 (2013).
[55] M. Pakmehr, B. D. McCombe, O. Chiatti, S. Buchholz, S. Fischer, C. Heyn, and W. Hansen, An Oral Presentation of the EP2DS-20 Conference 2013, ThP140 (2013).

[56] Q. Zhang, T. Arikawa, E. Kato, J. L. Reno, W. Pan, J. D. Watson, M. J. Manfra, M. A. Zudov, M. Tokman, M. Erukhimova, A. Belyanin, and J. Kono, Phys. Rev. Lett. 113, 047601 (2014).

[57] S. A. Mikhailov, Phys. Rev. B 70, 165311 (2004).

[58] S. D. Ganichev, V. V. Bel'kov, S. A. Tarasenko, S. N. Danilov, S. Giglberger, Ch. Hoffmann, E. L. Ivchenko, D. Weiss, W. Wegscheider, Ch. Gerl, D. Schuh, J. Stahl, J. De Boeck, G. Borghs, and W. Prettl, Nat. Phys. 2, 609 (2006).

[59] S. D. Ganichev, S. N. Danilov, V. V. Bel'kov, S. Giglberger, S. A. Tarasenko, E. L. Ivchenko, D. Weiss, W. Jantsch, F. Schäffler, D. Gruber, and W. Prettl, Phys. Rev. B 75, 155317 (2007).

[60] E. L. Ivchenko and G. E. Pikus, Izv. Akad. Nauk SSSR, Ser. Fiz. 47, 2369 (1983) [Bull. Acad. Sci. USSR, Phys. Ser. 47, 81 (1983)].

[61] V. I. Belinicher, Sov. Phys. - Solid State 24, 7 (1981).

[62] S. Titeica, Ann. Phys. 414, 129 (1935).

[63] M. G. Vavilov and I. L. Aleiner, Phys. Rev. B 69, 035303 (2004).

[64] I. A. Dmitriev, A. D. Mirlin, D. G. Polyakov, and M. A. Zudov, Rev. Mod. Phys. 84, 1709 (2012).

[65] We note that studied (013)-grown QWs belong to the $C_{1}$ point group. The general form of the spin-dependent matrix element is $V_{\boldsymbol{k}^{\prime} \boldsymbol{k}}=V_{0}+\sum_{\alpha \beta} V_{\alpha \beta} \sigma_{\alpha}\left(k_{\beta}+k_{\beta}^{\prime}\right)$ and the symmetry does not impose any restrictions on the components of second-rank pseudotensor $V_{\alpha \beta}$. Consequently, $\sigma_{z} k_{y}$ terms become possible and $y$ is some axis in the quantum well plane, whose direction may vary with the temperature, magnetic field, etc.

[66] S. D. Ganichev, S. A. Tarasenko, V. V. Bel'kov, P. Olbrich, W. Eder, D. R. Yakovlev, V. Kolkovsky, W. Zaleszczyk, G. Karczewski, T. Wojtowicz, and D. Weiss, Phys. Rev. Lett. 102, 156602 (2009).

[67] S. D. Ganichev and L. E. Golub, Phys. Status Solidi B 251, 1801 (2014).

[68] A. A. Abrikosov, Fundamentals of the Theory of Metals (NorthHolland, Amsterdam, 1988).

[69] J. Schliemann, New J. Phys. 10, 043024 (2008).

[70] At low temperatures the efficiency of electron-electron collisions scales as $\left(k_{B} T / E_{F}\right)^{2}$, see Refs. [71-73], where $E_{F}$ is the Fermi energy, while the temperature-induced suppression of quantum oscillations is governed by the factor $\left(k_{B} T / \hbar \omega_{c}\right)^{2}$.

[71] A. V. Chaplik, Sov. Phys. - JETP 33, 997 (1971).

[72] C. Hodges, H. Smith, and J. W. Wilkins, Phys. Rev. B 4, 302 (1971).

[73] G. F. Giuliani and J. J. Quinn, Phys. Rev. B 26, 4421 (1982).

[74] It is proportional to the product $\xi \sin 2 \theta$, where $\xi$ is the scattering asymmetry parameter introduced in Ref. [29] and $\theta$ is the angle between [001] and [013] axes.

[75] E. M. Lifshitz and L. P. Pitaevskii, Physical Kinetics (Butterworth-Heinemann, Oxford, 1981).

[76] Yu. A. Bychkov, Sov. Phys. - JETP 12, 971 (1961).

[77] T. Ando, J. Phys. Soc. Jpn. 37, 1233 (1974).

[78] N. S. Averkiev, L. E. Golub, S. A. Tarasenko, and M. Willander, J. Phys.: Condens. Matter 13, 2517 (2001).

[79] S. A. Tarasenko, Phys. Solid State 44, 1769 (2002).

[80] I. A. Dmitriev, A. D. Mirlin, and D. G. Polyakov, Phys. Rev. Lett. 91, 226802 (2003).

[81] O. E. Raichev, Phys. Rev. B 78, 125304 (2008). 
[82] Additional (radiative) broadening of the CR discussed in Refs. [56,57] is neglected hereafter.

[83] T. Ando, J. Phys. Soc. Jpn. 38, 989 (1975).

[84] S. G. Sharapov, V. P. Gusynin, and H. Beck, Phys. Rev. B 69, 075104 (2004).

[85] Equations (15) and (20) correct the misprints which are present in the corresponding equations of Ref. [40].

[86] Slight nonparabolicity of energy dispersion in these samples caused by the small value of the band gap results in a weak dependence of the CR position on the electron gas density; cf. Ref. [27].

[87] X. C. Zhang, K. Ortner, A. Pfeuffer-Jeschke, C. R. Becker, and G. Landwehr, Phys. Rev. B 69, 115340 (2004).

[88] We note that the 21-nm sample has a nontrivial band structure where indirect interband optical transitions as well as semimetallic behavior are possible [89]. However, in our experiments the Fermi energy in this sample, being on the order of $15 \mathrm{meV}$, exceeds the energy of radiation quantum $(10 \mathrm{meV})$, therefore, at least at low temperatures, the radiation absorption is mostly governed by the indirect intraband transitions (Drude absorption). Thus, the mechanisms of the photocurrent generation for this wide quantum well remain the same as for other samples.

[89] Z. D. Kvon, E. B. Olshanetsky, D. A. Kozlov, N. N. Mikhailov, and S. A. Dvoretsky, JETP Lett. 87, 502 (2008); E. B. Olshanetsky, Z. D. Kvon, N. N. Mikhailov, E. G. Novik, I. O. Parm, and S. A. Dvoretsky, Solid State Commun. 152, 265 (2012).
[90] We note that the photovoltaic effect in field-effect transistors is also known to demonstrate quantum $1 / B$-periodic magnetooscillations enhanced at the CR [91-93], however, it is described by the different microscopic mechanism related with the plasma waves excitation in gated structures and, by contrast to $j_{n}$, is insensitive to the spin polarization.

[91] M. B. Lifshits and M. I. Dyakonov, Phys. Rev. B 80, 121304(R) (2009).

[92] S. Boubanga-Tombet, M. Sakowicz, D. Coquillat, F. Teppe, W. Knap, M. I. Dyakonov, K. Karpierz, J. Lusakowski, and M. Grynberg, Appl. Phys. Lett. 95, 072106 (2009).

[93] O. A. Klimenko, Yu. A. Mityagin, H. Videlier, F. Teppe, N. V. Dyakonova, C. Consejo, S. Bollaert, V. N. Murzin, and W. Knap, Appl. Phys. Lett. 97, 022111 (2010).

[94] V. V. Bel'kov and S. D. Ganichev, in Handbook of Spintronic Semiconductors, edited by W. M. Chen and I. A. Buyanova (Pan Stanford, Singapore, 2010), pp. 243-265.

[95] V. Lechner, L. E. Golub, P. Olbrich, S. Stachel, D. Schuh, W. Wegscheider, V. V. Bel'kov, and S. D. Ganichevand, Appl. Phys. Lett. 94, 242109 (2009).

[96] For the long-range scattering potential where $\tau_{q} \neq \tau_{\text {tr }}$ and in the presence of nonparabolicity of electron energy spectrum, additional contributions to $j_{\mu}$ may arise due to the energy dependence of the scattering times and effective mass, which are neglected in our consideration. These contributions may only provide a smooth background of the photocurrent superimposed by oscillations and are small by the parameter $\Delta_{Z} / E_{F}$. 